\title{
AUGER RECOMBINATION IN SEMICONDUCTOR QUANTUM WELLS
}

\author{
Anatoli S. Polkovnikov and Georgy G. Zegrya \\ Ioffe Physico-Technical Institute \\ Russia, 194021, St.-Petersburg, Politechnicheskaya st. 26
}

\begin{abstract}
The principal mechanisms of Auger recombination of nonequilibrium carriers in semiconductor heterostructures with quantum wells are investigated. It is shown for the first time that there exist three fundamentally different Auger recombination mechanisms of (i) thresholdless, (ii) quasi-threshold, and (iii) threshold types. The rate of the thresholdless Auger process depends on temperature only slightly. The rate of the quasi-threshold Auger process depends on temperature exponentially. However, its threshold energy essentially varies with quantum well width and is close to zero for narrow quantum wells. It is shown that the thresholdless and the quasi-threshold Auger processes dominate in narrow quantum wells, while the threshold and the quasi-threshold processes prevail in wide quantum wells. The limiting case of a three-dimensional (3D) Auger process is reached for infinitely wide quantum wells. The critical quantum well width is found at which the quasi-threshold and threshold Auger processes merge into a single 3D Auger process. Also studied is phonon-assisted Auger recombination in quantum wells. It is shown that for narrow quantum wells the act of phonon emission becomes resonant, which in turn increases substantially the coefficient of phonon-assisted Auger recombination. Conditions are found under which the direct Auger process dominates over the phonon-assisted Auger recombination at various temperatures and quantum well widths.
\end{abstract}

\section{INTRODUCTION}

Two recombination processes are predominant in semiconductors at high excitation levels: (i) radiative recombination and (ii) nonradiative Auger recombination related to the electronelectron interaction. For homogeneous semiconductors, mechanisms of Auger recombination (AR) have been extensively studied回-日. In narrow gap semiconductors occur AR processes 
involving two electrons and a heavy hole (CHCC Auger process) or an electron and two heavy holes, with transition of one of the holes to the SO zone (CHHS Auger process)2 2. B. Both these processes are of threshold nature, and the rate of Auger recombination changes with temperature exponentially 国. The only exception are semiconductors in which the spin-orbit splitting is close to the energy gap ( GaSb and InAs). Under certain conditions the rate of the CHHS process in these semiconductors depends on temperature only slightly $\mathrm{\theta}$. It is commonly believed that in weakly doped semiconductors phonon-assisted AR processes are predominant at low temperature and high excitation levels 3 . B. Because of the large momentum transferred to a phonon, the threshold for heavy holes is removed and phonon-assisted Auger process becomes a power-law function of temperature.

Single semiconductor heterostructures, quantum wells, quantum wires, quantum dots are spatially inhomogeneous owing to the existence of barriers. The presence of a heteroboundary affects not only the energy and wave functions of carriers, but also the macroscopic properties of heterostructures 7 , which is of primary importance. It is commonly believed that the AR mechanism in quantum wells is the same as in a homogeneous semiconductor 1 月 1 - Nevertheless, the presence of a heteroboundary strongly affects the electron-electron interaction in quantum wells, and this influence is of fundamental nature. The heteroboundary lifts restrictions imposed on processes of electron-electron interaction by the energy and momentum conservation laws. Namely, the conservation of quasi-momentum perpendicular to the heteroboundary breaks down. In turn, this leads to the appearance in heterostructures of new thresholdless channels of Auger recombinationd. The rate of the thresholdless AR process is a power function of temperature. The first direct experiment aimed at observing the thresholdless AR channel at $T=77 \mathrm{~K}$ was reported in 12 . At low temperature the thresholdless process, in contrast to that with a threshold, is rather an efficient channel of nonradiative recombination, and for narrow quantum wells and high concentrations it dominates the phonon-assisted AR process. The existence of a thresholdless matrix element of electron-electron interaction also exerts strong influence on the phonon-assisted AR process. The latter becomes resonant and is much enhanced as compared with the 3D phonon-assisted Auger process.

A detailed analysis of the threshold and thresholdless AR mechanisms has been performed for a single heterobarriert. Conditions were studied under which the thresholdless channel dominates over the threshold one. For quantum wells no such detailed analysis has been done. Taylor et al 13 considered the possibility of removing the threshold for AR in quantum wells upon transition of excited carriers to the continuos part of spectrum. However, no microscopic theory of the thresholdless process was given in this work and no theoretical analysis performed of the competition between the threshold, quasi-threshold, and thresholdless AR mechanisms at various temperatures and quantum well widths. Only the thresholdless AR channel, cor- 
responding to small momenta transferred in Coulomb interaction of particles (for the CHCC process) with spin-orbit interaction neglected, was considered in 14.15.

The aim of the present work is to investigate theoretically the principal mechanisms of AR for nonequilibrium carriers in semiconductor quantum wells. It will be shown that there exist three fundamentally different AR mechanisms: (i) threshold mechanism similar to an Auger process in a homogeneous semiconductor, (ii) quasi-threshold mechanism with a threshold energy strongly depending on the quantum well width, and (iii) thresholdless mechanism inoperative in a homogeneous semiconductor. For the threshold AR process in a quantum well the threshold energy is close to that in a homogeneous semiconductor. Conversely, owing to the small value of the threshold energy, the rate of the quasi-threshold process in narrow quantum wells depends on temperature only slightly. For this reason there is no clear distinction between mechanisms (ii) and (iii) in sufficiently narrow quantum wells, and they may be considered as a single thresholdless AR process. With increasing quantum well width, the threshold energy of the quasi-threshold process increases and approaches the bulk value. A totally different behavior is characteristic of the thresholdless AR mechanism. With increasing quantum well width, its rate falls dramatically and, on passing to a homogeneous semiconductor, this mechanism ceases to be operative. Conditions will be found under which the thresholdless AR mechanism dominates the threshold one. The critical quantum well width will be found, at which the quasi-threshold and the threshold Auger processes merge and form a single 3D AR process. Also, the phonon-assisted AR in quantum wells will be studied. It will be shown that for narrow quantum wells the act of phonon emission becomes resonant, which in turn increases substantially the coefficient of phonon-assisted AR. Conditions will be found under which the direct AR process dominates the phonon-assisted AR process at various temperatures and quantum well widths.

\section{PRINCIPAL EQUATIONS}

To analyze the AR mechanisms and find the rate of the Auger process, wave functions of charge carriers are to be known. As already established for bulk Auger processes, the wave functions of carriers must be calculated using the multiband approximation日. We will use the four-band Kane's model, the most adequately describing the wave functions and energy spectrum of carriers in narrow-gap $\mathrm{A}_{I I I} \mathrm{~B}_{V}$ semiconductorst 16 .

\section{Wave functions in a homogeneous semiconductor}

For most $\mathrm{A}_{I I I} \mathrm{~B}_{V}$ semiconductors, wave functions of electrons and holes in the center of the 
Brillouin zone are described by the $\Gamma_{6}^{+}$representation for the conduction band and by the $\Gamma_{7}^{+}$ and $\Gamma_{8}^{+}$representations for the valence band. Of these the first two and the last are doubly and fourfold degenerate, respectively. The corresponding equations for wave functions may be written in differential form. Commonly, the basis wave functions of the conduction and valence bands are taken in form of eigenfunctions of the angular momentum 6 日. 0 . However, another representation of the basis functions is more appropriate for our purposes:

$$
|s \uparrow\rangle,|s \downarrow\rangle,|x \uparrow\rangle,|x \downarrow\rangle,|y \uparrow\rangle,|y \downarrow\rangle,|z \uparrow\rangle,|z \downarrow\rangle,
$$

where $|s\rangle$ and $|x\rangle,|y\rangle,|z\rangle$ are the Bloch functions of $s$ and $\mathbf{p}$ type with angular momenta of 0 and 1, respectively. The former describe the state of the conduction band and the latter the state of the valence band at the $\Gamma$-point. Arrows denote the direction of spin. The wave function of carriers $\psi$ may be presented in the form:

$$
\psi=\Psi_{s}|s\rangle+\Psi|\mathbf{p}\rangle,
$$

where $\Psi_{s}$ and $\Psi$ are spinors. In the vicinity of the $\Gamma$-point the equations for $\Psi_{s}$ and $\Psi$ envelopes written in the spherical approximation are as follows:

$$
\left\{\begin{array}{l}
\left(E_{c}-E\right) \Psi_{s}-i \hbar \gamma \nabla \boldsymbol{\Psi}=0, \\
\left(E_{v}-\delta-E\right) \boldsymbol{\Psi}-i \hbar \gamma \nabla \Psi_{s}+\frac{\hbar^{2}}{2 m}\left(\tilde{\gamma}_{1}+4 \tilde{\gamma}_{2}\right) \nabla(\nabla \boldsymbol{\Psi})- \\
-\frac{\hbar^{2}}{2 m}\left(\tilde{\gamma}_{1}-2 \tilde{\gamma}_{2}\right) \nabla \times[\nabla \times \boldsymbol{\Psi}]+i \delta[\boldsymbol{\sigma} \times \boldsymbol{\Psi}]=0 .
\end{array}\right.
$$

Here $\gamma$ is the Kane's matrix element 17 having dimension of velocity, $\widetilde{\gamma}_{1}$ and $\widetilde{\gamma}_{2}=\widetilde{\gamma}_{3}$ are the generalized Luttinger parameter $177, \delta=\Delta_{s o} / 3, \Delta_{s o}$ is the spin orbit splitting, $E_{v}$ and $E_{c}$ are the energies of the lower edge of the conduction band and the upper edge of the valence band, $m$ is the free-electron mass, $\boldsymbol{\sigma}=\left(\sigma_{x}, \sigma_{y}, \sigma_{z}\right)$ are the Pauli spin matrices. If, instead of using the Luttinger parameters, the heavy hole mass describing the interaction with higher bands is introduced phenomenologically, then equations (2) are transformed into equations derived by

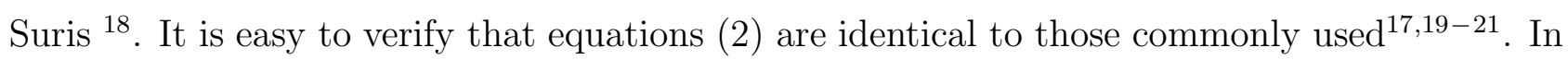
the first equation in the system (2) we neglect the term with mass of free electrons.

\section{A. Hole states}

The expression for $\Psi_{s}$ can be found from the first equation of the system (21). Substitution of $\Psi_{s}$ into the second equation gives:

$$
-E \boldsymbol{\Psi}+\frac{\hbar^{2}}{2 m_{l}} \nabla(\nabla \boldsymbol{\Psi})-\frac{\hbar^{2}}{2 m_{h}} \nabla \times[\nabla \times \boldsymbol{\Psi}]+i \delta[\boldsymbol{\sigma} \times \mathbf{\Psi}]=0,
$$

where 


$$
m_{l}^{-1}=\frac{2 \gamma^{2}}{E_{g}+\delta-E}+m^{-1}\left(\widetilde{\gamma}_{1}+4 \widetilde{\gamma}_{2}\right), \quad m_{h}^{-1}=m^{-1}\left(\widetilde{\gamma}_{1}-2 \widetilde{\gamma}_{2}\right)
$$

Here $m_{h}$ coincides with the heavy hole mass, and $m_{l}$ with the light hole mass in the case of zero constant of spin-orbit interaction; $E_{g}=E_{c}-E_{v}$ is the semiconductor forbidden gap. For the sake of convenience, it is assumed that $E_{v}=\delta$. This choice is due to an increase in the heavy hole and light hole energies at the $\Gamma$-point by $\delta$ and a decrease in the spin split off $(\mathrm{SO})$ hole energy by $2 \delta$ under the action of spin-orbital interaction (see eq. 7). Equation (3) can be simplified by introducing new functions

$$
\phi=\operatorname{div} \boldsymbol{\Psi} \text { and } \eta=\boldsymbol{\sigma} \operatorname{rot} \boldsymbol{\Psi}
$$

After taking the divergence and rotor of equation (3), multiplied by $\sigma$ it is transformed into a system of two differential equations

$$
\left\{\begin{array}{l}
-E \phi+\frac{\hbar^{2}}{2 m_{l}} \Delta \phi+i \delta \eta=0 \\
-(E+\delta) \eta+\frac{\hbar^{2}}{2 m_{h}} \Delta \eta-2 i \delta \phi=0
\end{array}\right.
$$

Fourier transform of these equations gives hole spectra for a homogeneous semiconductor

$$
\left[\begin{array}{cc}
E+\frac{\hbar^{2}}{2 m_{l}} k^{2} & i \delta \\
-2 i \delta & E+\frac{\hbar^{2}}{2 m_{h}} k^{2}+\delta
\end{array}\right]\left(\begin{array}{l}
\phi \\
\eta
\end{array}\right)=0
$$

The characteristic equation has two roots

$$
E_{1,2}=-\frac{\delta}{2}-\frac{\hbar^{2} k^{2}}{4}\left(m_{l}^{-1}+m_{h}^{-1}\right) \pm \sqrt{2 \delta^{2}+\left(\frac{\delta}{2}-\frac{\hbar^{2} k^{2}}{4}\left(m_{l}^{-1}-m_{h}^{-1}\right)\right)^{2}}
$$

It should be noted that $m_{l}$ depends on energy (see eq. (3)). At the $\Gamma$-point $(k=0)$ we have the roots $E_{1}=\delta$ and $E_{2}=-2 \delta$. The positive solution corresponds to light holes, and that with negative sign to $\mathrm{SO}$ holes.

In the vicinity of the $\Gamma$-point the energies $E_{1,2}$ can be expanded into a series in terms of wave vector to relate the effective light and SO-hole masses $m_{h l}, m_{s o}$ and the Luttinger parameters:

$$
E_{1} \approx \delta-\frac{\hbar^{2} k^{2}}{2 m_{h l}}, \quad E_{2} \approx-2 \delta-\frac{\hbar^{2} k^{2}}{2 m_{s o}}
$$

where

$$
m_{h l}^{-1}=\frac{4 \gamma^{2}}{3 E_{g}}+\frac{\left(\tilde{\gamma}_{1}+2 \tilde{\gamma}_{2}\right)}{m}, \quad m_{s o}^{-1}=\frac{2 \gamma^{2}}{3\left(E_{g}+3 \delta\right)}+\frac{\tilde{\gamma}_{1}}{m} .
$$

An approximate light hole spectrum can be obtained by means of a widely used $4 \times 4$ Hamiltonian 20. However, the range of its applicability is rather narrow, since commonly $m_{l} \sim 0.1 m_{h}$ and the expansion (8) is only valid when $E \ll \frac{m_{l}}{m_{h}} \Delta_{s o}$. Moreover, such a model cannot describe 
Auger transitions at all, since the basis states of carriers in different bands are orthogonal. The same applies to the spectrum of the SO band.

The Fourier amplitudes of the wave functions of both light and SO holes can be presented in the form (see eq. (3)):

$$
\boldsymbol{\Psi}=\mathbf{k} f+\frac{i \delta}{E+\delta+\frac{\hbar^{2} k^{2}(E)}{2 m_{h}}}[\mathbf{k} \times \boldsymbol{\sigma} f], \quad \Psi_{s}=-\frac{\hbar \gamma k^{2}(E)}{E_{g}+\delta-E} f,
$$

where $f$ is an arbitrary spinor related to the previously introduced function $\phi$ by the expression $\phi=k^{2}(E) f$.

The third solution of (3) pertaining to heavy holes satisfies the relations $\operatorname{div} \boldsymbol{\Psi}=0$ (as a consequence $\Psi_{s}=0$ ) and $\boldsymbol{\sigma} \operatorname{rot} \Psi=0$. This follows from equation (5), since, if $\phi=0$, then $\eta=0$ and vice versa. It can be readily seen that

$$
\left[\boldsymbol{\sigma} \times \boldsymbol{\Psi}_{h}\right]=-i \boldsymbol{\Psi}_{h}
$$

Thus, the dispersion law describing the heavy hole spectrum looks like

$$
E_{h}=\delta-\frac{\hbar^{2} k_{h}^{2}}{2 m_{h}}
$$

The components of the heavy hole wave function must satisfy the equations:

$$
\begin{gathered}
\left\{\begin{array}{l}
\Psi_{z \downarrow}=\left(\Psi_{x \uparrow}+i \Psi_{y \uparrow}\right) \\
\Psi_{z \uparrow}=\left(-\Psi_{x \downarrow}+i \Psi_{y \downarrow}\right)
\end{array} \Leftrightarrow[\boldsymbol{\sigma} \times \boldsymbol{\Psi}]=-i \boldsymbol{\Psi},\right. \\
\left\{\begin{array}{l}
k_{z} \Psi_{z \uparrow}+k_{x} \Psi_{x \uparrow}+k_{y} \Psi_{y \uparrow}=0 \\
k_{z} \Psi_{z \downarrow}+k_{x} \Psi_{x \downarrow}+k_{y} \Psi_{y \downarrow}=0
\end{array} \Leftrightarrow \operatorname{div} \boldsymbol{\Psi}=0 .\right.
\end{gathered}
$$

Solving these equations one may obtain the explicit expressions for the wave functions. For a quantum well they are given in Appendix A.

\section{B. Electron states}

In principle, the conventional equations for electrons have the same form as those for holes. Since the $\Gamma$-point in the conduction band is only doubly degenerate, and the crystal field causes no additional splitting, there is no need to retain terms with parameters $\widetilde{\gamma}_{i}$. Moreover, the presence of these terms in the equations for electrons gives a far too exact model. Thus, a simplified model will be used for electrons 


$$
\left\{\begin{array}{l}
\left(E_{c}-E\right) \Psi_{s}-i \hbar \gamma \nabla \boldsymbol{\Psi}=0 \\
\left(E_{v}-\delta-E\right) \boldsymbol{\Psi}-i \hbar \gamma \nabla \Psi_{s}+i \delta[\boldsymbol{\sigma} \times \boldsymbol{\Psi}]=0
\end{array}\right.
$$

The electron energies can be conveniently reckoned from the lower edge of the conduction band $\left(E_{c}=0\right)$. This energy will be denoted by $\mathcal{E}$, so that it would not be confused with the full energy of electron $E$, reckoned from the same level as the hole energy. Introducing into equation (13) the functions $\phi$ and $\eta$ in the same form as before (see eq. (雨)), one can obtain

$$
\begin{aligned}
& -\left(E_{g}+\delta+\mathcal{E}\right) \phi+\frac{\hbar^{2} \gamma^{2}}{\mathcal{E}} \Delta \phi+i \delta \eta=0 \\
& -\left(E_{g}+\mathcal{E}+2 \delta\right) \eta-2 i \delta \phi=0
\end{aligned}
$$

Passing to a Fourier transform, we find the electron dispersion law

$$
k^{2}=\frac{\mathcal{E}}{\hbar^{2} \gamma^{2}} \frac{\mathcal{E}^{2}+\mathcal{E}\left(2 E_{g}+3 \delta\right)+\left(E_{g}+3 \delta\right) E_{g}}{E_{g}+\mathcal{E}+2 \delta} .
$$

If $\mathcal{E} \ll E_{g}, \delta$, then the energy is quadratic in wave vector.

$$
\mathcal{E}=\frac{\hbar^{2} k^{2}}{2 m_{c}}
$$

where

$$
m_{c}^{-1}=2 \gamma^{2} \frac{E_{g}+2 \delta}{\left(E_{g}+3 \delta\right) E_{g}} .
$$

The Fourier amplitude of the wave function is given by

$$
\Psi_{s}=f, \quad \Psi=\frac{\mathcal{E}}{\hbar \gamma k^{2}(\mathcal{E})}\left[\mathbf{k} f+\frac{i \delta}{\mathcal{E}+E_{g}+2 \delta}[\mathbf{k} \times(\boldsymbol{\sigma} f)]\right],
$$

where $f$ is an arbitrary spinor (see eq. (9)).

C. Probability flux and the equations near the heteroboundary

An expression for the probability flux density can be derived from equation (2) by substituting $E \rightarrow-i \hbar \frac{\partial}{\partial t}$ and using then a procedure similar to that employed in quantum mechanics 22 . It can also be derived by the $\mathbf{k p}$ method in the second-order perturbation theory. As a result, the following expression is obtained in the case of holes for the probability flux density

$$
\mathbf{j}_{h}=\frac{E_{g}+\delta-E}{2 m_{l} \gamma}\left[\Psi_{s} \boldsymbol{\Psi}^{*}+\Psi_{s}^{*} \boldsymbol{\Psi}\right]-\frac{i \hbar}{2 m_{h}}\left(\boldsymbol{\Psi} \times \operatorname{rot} \boldsymbol{\Psi}^{*}-\boldsymbol{\Psi}^{*} \times \operatorname{rot} \boldsymbol{\Psi}\right) .
$$

For electrons in the conduction band this expression takes a simpler form 


$$
\mathbf{j}_{e}=\gamma\left[\Psi_{s} \Psi^{*}+\Psi_{s}^{*} \Psi\right]
$$

The exact procedure for deriving boundary conditions for wave functions at the heteroboundary still remains to be devised. However, some approximate methods for solving this problem have been developed in recent years. The Kane's parameter $\gamma$ usually differs only slightly for $A_{I I I} B_{V}$ semiconductors, hence continuity of $\gamma$ is usually supposed in literature (see for example 17 ). Discrepancy of parameter $\gamma$ in a quantum well and barrier region results in small change of Auger coefficient (see section IV). Following the method elaborated by Burt 19 and asuming contunuity of Kane's parameter, we derive from the system (2) Kane's equations which can be integrated across the heterobarrier:

$$
\left\{\begin{array}{l}
\left(E_{g}+\delta-E\right) \Psi_{s}-i \hbar \gamma \nabla \boldsymbol{\Psi}=0, \\
-E \boldsymbol{\Psi}-i \hbar \gamma \nabla \Psi_{s}+\frac{\hbar^{2}}{2 m} \nabla\left[6 \tilde{\gamma}_{2} \nabla \boldsymbol{\Psi}\right]+ \\
+\frac{\hbar^{2}}{2 m} \frac{\partial}{\partial x_{k}}\left(\tilde{\gamma}_{1}-2 \tilde{\gamma}_{2}\right) \frac{\partial}{\partial x_{k}} \boldsymbol{\Psi}+i \delta[\boldsymbol{\sigma} \times \boldsymbol{\Psi}]=0 .
\end{array}\right.
$$

Using these equations and the probability flux density conservation law we derive the boundary conditions for the wave-function envelopes.

\section{Carrier states in a quantum well}

The wave functions of carriers in a quantum well may be derived using the symmetry properties of the Hamiltonian. Spinless Hamiltonian $\mathcal{H}_{0}$ is invariant with respect to the substitution $x \rightarrow-x$. Consider an operator $\mathcal{R}$ such that

$$
\begin{gathered}
\mathcal{R}:(x, y, z) \rightarrow(-x, y, z), \quad \mathcal{R}=\mathcal{I C}_{\pi x}, \\
\mathcal{H}_{0} \mathcal{R}=\mathcal{R H}_{0}
\end{gathered}
$$

where $\mathcal{I}$ is the inversion operator, and $\mathcal{C}_{\pi x}$ is the operator of rotation by an angle $\pi$ around the $x$ axis perpendicular to the plane of the quantum well.

With account of the spin orbit interaction the Hamiltonian can be written in the form:

$$
\mathcal{H}=\mathcal{H}_{0}+\frac{\hbar}{4 m^{2} c^{2}}[\nabla V \times \mathbf{p}] \boldsymbol{\sigma}
$$

where $\mathbf{p}$ is the momentum operator and $V$ is the potential energy of an electron in the crystal. The last term does not commute with $\mathrm{R}$. Therefore, the symmetry operator $\mathcal{D}$ may be sought for as a product of operator $\mathcal{R}$ and some spin matrix $S$ to be found: $\mathcal{D}=\mathcal{R} \otimes S$. Since inversion leaves unchanged the sign of the vector product, the matrix $S$ must satisfy the relations 


$$
\left\{\begin{array}{l}
S \sigma_{x}=\sigma_{x} S \\
S \sigma_{y}=-\sigma_{y} S \\
S \sigma_{z}=-\sigma_{z} S
\end{array}, \sigma_{x}=\left[\begin{array}{ll}
0 & 1 \\
1 & 0
\end{array}\right], \sigma_{y}=\left[\begin{array}{cc}
0 & -i \\
i & 0
\end{array}\right], \sigma_{z}=\left[\begin{array}{cc}
1 & 0 \\
0 & -1
\end{array}\right] .\right.
$$

Obviously a Pauli spin matrix $\sigma_{x}$ may be taken for the matrix $S: S=\sigma_{x}$.

The functions $\Psi(x, y, z)$ and $\mathcal{D} \Psi(-x, y, z)$ satisfy the same equation. For this reason the eigenfunctions of the Hamiltonian may be sought for as eigenfunctions of the operator $\mathcal{D}$.

$$
\Psi(x, y, z)=\nu \mathcal{D} \Psi(-x, y, z), \text { where } \nu= \pm 1
$$

The values $\nu= \pm 1$ correspond to carrier states with different symmetry. With the wave functions chosen in such a way, the boundary conditions may be satisfied at one heteroboundary only, since at the other they will be fulfilled automatically. Solving equation (24) we find the necessary conditions for various components of the symmetrized wave function.

$$
\begin{array}{r}
\Psi_{s \uparrow}(x, y, z)= \pm \Psi_{s \downarrow}(-x, y, z), \Psi_{x \uparrow}(x, y, z)=\mp \Psi_{x \downarrow}(-x, y, z), \\
\Psi_{y \uparrow}(x, y, z)= \pm \Psi_{y \downarrow}(-x, y, z), \Psi_{z \uparrow}(x, y, z)= \pm \Psi_{z \downarrow}(-x, y, z) \\
\text { where the sign } "+" \text { corresponds to } \nu=1 \text {, and } "-\text { " to } \nu=-1
\end{array}
$$

Corresponding expressions for the components of electron and hole wave functions are given in Appendix A.

\section{PROBABILITY OF AUGER RECOMBINATION}

The probability of AR per unit time can be calculated in terms of the first-order perturbation theory in electron-electron interaction:

$$
W_{i \rightarrow f}=\frac{2 \pi}{\hbar}\left|M_{f i}\right|^{2} \delta\left(\varepsilon_{f}-\varepsilon_{i}\right)
$$

where

$$
M_{f i}=\left\langle\Psi_{f}\left(\boldsymbol{r}_{1}, \boldsymbol{r}_{2}, \nu_{1}, \nu_{2}\right)\left|\frac{e^{2}}{\kappa_{0}\left|\boldsymbol{r}_{1}-\boldsymbol{r}_{2}\right|}+\tilde{\Phi}\left(\mathbf{r}_{1}, \mathbf{r}_{2}\right)\right| \Psi_{i}\left(\boldsymbol{r}_{1}, \boldsymbol{r}_{2}, \nu_{1}, \nu_{2}\right)\right\rangle
$$

is the matrix element of electron-electron interaction, $\boldsymbol{r}_{1}$ and $\boldsymbol{r}_{2}$ are carrier coordinates, $\nu_{1}$ and $\nu_{2}$ are spin variables (see eq. (24)), $e$ is an electron charge, and $\kappa_{0}$ is the dielectric constant of the intrinsic semiconductor, $\tilde{\Phi}\left(\mathbf{r}_{1}, \mathbf{r}_{2}\right)$ is the additional potential arising because of difference between quantum well and barrier dielectric constants. The explicit expressions for $\tilde{\Phi}\left(\mathbf{r}_{1}, \mathbf{r}_{2}\right)$ are given in the Appendix B.

Taking into account the antisymmetrized form of the wave functions, the matrix element of Auger transition is the following: 


$$
M_{f i}=M_{\mathrm{I}}-M_{\mathrm{II}},
$$

where

$$
M_{\mathrm{I}}=\left\langle\Psi_{3}\left(\boldsymbol{r}_{1}, \nu_{1}\right) \Psi_{4}\left(\boldsymbol{r}_{2}, \nu_{2}\right)\left|\frac{e^{2}}{\kappa_{0}\left|\boldsymbol{r}_{1}-\boldsymbol{r}_{2}\right|}+\tilde{\Phi}\left(\mathbf{r}_{1}, \mathbf{r}_{2}\right)\right| \Psi_{1}\left(\boldsymbol{r}_{1}, \nu_{1}\right) \Psi_{2}\left(\boldsymbol{r}_{2}, \nu_{2}\right)\right\rangle,
$$

the expression for $M_{I I}$ may be derived from (29) by swaping indexes 1 and 2 in the wave functions $\Psi_{1}$ and $\Psi_{2}$. Hereafter the indexes $I$ and $I I$ in the expressions for the matrix elements will be omitted.

We shall consider two AR processes, CHCC and CHHS, since in fact only these two determine the rate of Auger recombination. Strictly speaking such a terminology is inapplicable to carriers in a quantum well, since there exists mixing between heavy hole, light hole and SO hole subbands. However, as noted above, in the case $m_{c} \ll m_{h}$ the extent of mixing between heavy and light holes is low, and the mixing of SO holes with heavy and light holes is negligible at $\Delta_{s o} \gg T$. The last condition is nearly always fulfilled for $\mathrm{A}_{I I I} \mathrm{~B}_{V}$ semiconductors. For this reason we may rely on the above terminology.

\section{Matrix element of Auger transition}

Evaluations of matrix elements for the $\mathrm{CHCC}$ and CHHS Auger processes are similar. For the sake of simplicity later in this section the matrix element of the CHCC Auger transition will be mainly discussed. However, in the following section approximate expressions for the Auger coefficient will be given both for the CHCC and for the CHHS processes. The matrix element of an electron-electron Coulomb interaction can be most conveniently calculated using a Fourier transform. We take into account that the wave functions of carriers in a quantum well are the plane waves along the lateral direction:

$$
\Psi_{i}(\mathbf{r})=\psi_{i}\left(x, \mathbf{q}_{i}\right) e^{i \mathbf{q}_{i} \rho} .
$$

The explicit expressions for wave functions of electrons and holes $\psi_{i}$ are given in the Appendix A. Then

$$
\begin{aligned}
M & =\frac{4 \pi e^{2}}{\kappa_{0}} \frac{1}{2 q} \int_{-\infty}^{\infty} \int_{-a / 2}^{a / 2} \psi_{4}^{*}\left(x_{1}\right) \psi_{3}^{*}\left(x_{2}\right) \times \\
& \times\left(e^{-q\left|x_{1}-x_{2}\right|}+\tilde{\phi}\left(x_{1}, x_{2}, q\right)\right) \psi_{1}\left(x_{1}\right) \psi_{2}\left(x_{2}\right) d x_{1} d x_{2},
\end{aligned}
$$

$q=\left|q_{1}-q_{4}\right|=\left|q_{3}-q_{2}\right|$ is the momentum transferred in the plane of the quantum well in Coulomb interaction, $a$ is the quantum well width $\tilde{\phi}$ corresponds to the potential $\tilde{\Phi}$ and the expression for it is given in the Appendix B. The integrating over $x_{2}$ is limited within the quantum well due to the fact that heavy holes, because of their relatively big mass, are usually 
strongly localized inside the well. Hereafter $x$ denotes the coordinate orthogonal to the quantum well plain and $\boldsymbol{\rho}$ denotes the pair of coordinates in the quantum well plane, $\mathbf{q}$ and $k$ are the lateral and $x$ - quasimomentum components of particles.

As it is seen from the equation (30) the Auger scattering occurs on the one-dimensional exponentially decaying potential which depends on the lateral transferred momentum. The state of the excited particle may lie in both the continuous and discrete spectrum, W $_{\text {with }}$ the latter situation occurring when the longitudinal momentum of the particle much exceeds the transverse momentum. In determining the rate of Auger recombination, both localized and delocalized states must be considered as final states of the excited particle. Possibility of an electron (hole) transition into a localized or into a free state leads to the existence of different AR mechanisms in quantum wells.

Calculation of the matrix element of Auger recombination for a transition of the excited particle into the continuous spectrum.

For evaluating the matrix element we use the approximation

$$
V_{c}, V_{v} \ll E_{g},
$$

where $V_{c}$ and $V_{v}$ are the barrier heights for electrons and holes respectively. Obviously, this approximation also implies that $k_{4}^{2}+q^{2} \gg k_{1}^{2}$, i.e. the total momentum of the excited electron is much larger than that of the localized one. Integral over $x_{1}$ coordinate can be found by integrating by parts. The $\mathrm{n}$-th antiderivative of the function $\psi_{4} e^{-q x}$ is:

$$
F_{4}^{n}(q, x)=(-1)^{n} \frac{\left(e^{q x} \psi_{4}(x)\right)^{(n)}}{\left(k_{4}^{2}+q^{2}\right)^{n}} e^{-2 q x} .
$$

Then the approximate expression for the matrix element $M_{I}$ may be obtained:

$$
M \approx M^{(1)}+M^{(2)},
$$

where

$$
\begin{aligned}
M^{(1)} & =-\frac{4 \pi e^{2}}{\kappa_{0}\left(q^{2}+k_{4}^{2}\right)}\left(\mathcal{F}(a / 2) \int_{-a / 2}^{a / 2} e^{q x_{2}} \psi_{3}^{*}\left(x_{2}\right) \psi_{2}\left(x_{2}\right) d x_{2}-\right. \\
& \left.-\mathcal{F}(-a / 2) \int_{-a / 2}^{a / 2} e^{-q x_{2}} \psi_{3}^{*}\left(x_{2}\right) \psi_{2}\left(x_{2}\right) d x_{2}\right) .
\end{aligned}
$$

Here 


$$
\mathcal{F}(a / 2)=e^{-q a / 2} \psi_{4 s}^{*}(a / 2) \psi_{1 s}(a / 2)\left(\frac{3 V_{c}+V_{v}}{4 E_{g}}-\frac{\kappa_{0}-\tilde{\kappa}_{0}}{\kappa_{0}+\tilde{\kappa}_{0}}\right) .
$$

Index $s$ in the $\psi_{4 s}$ and $\psi_{1} 1 s$ implies that only $s$-components of the wave functions are taken, $\tilde{\kappa}_{0}$ is the dielectric constant in the barrier region.

$$
M^{(2)}=\frac{4 \pi e^{2}}{\kappa_{0}\left(q^{2}+k_{4}^{2}\right)} \int_{-a / 2}^{a / 2} \psi_{4}^{*}(x) \psi_{3}^{*}(x) \psi_{2}(x) \psi_{1}(x) d x
$$

Note, as the wave functions are spinors the components of $\psi_{4}^{*}$ should be multiplied by components of $\psi_{1}$ and vice versa the components of $\psi_{3}^{*}$ should be multiplied by those of $\psi_{2}$.

In this way it appears that the matrix element of Auger transition splits two parts. The first of them is related to the presence of heterobundaries and the second one corresponds to the short range Coulomb scattering. The latter can be easily understood as during Auger transition a large momentum is transmitted from the localized electron to the excited one and this is possible only if the scattering particles find themselves very close to each other. Note that both $M^{(1)}$ and $M^{(2)}$ and, consequently, the matrix element $M$ itself are in fact thresholdless matrix elements. Indeed, they are not subject to any restrictions imposed on the initial momenta of carriers, $k_{1}, k_{c}, k_{h}$. However, the mechanisms responsible for the momentum nonconservation $k_{1}+k_{2} \neq k_{3}+k_{4}$ in the components $M^{(1)}$ and $M^{(2)}$ are different. In $M^{(1)}$ the nonconservation is related to carrier scattering at the heteroboundary, and the same mechanism gives rise to a thresholdless Auger process in scattering on a single heterobarrier 7 . The reason why the conservation law breaks down for $M^{(2)}$ is that the volume of integration with respect to $x$ is restricted to the quantum well region, which results in the appearance of a function of the type $\frac{\sin k a / 2}{k}$ instead of $\delta(k)$. The physical meaning of the above distinctions between the matrix elements $M^{(1)}$ and $M^{(2)}$ is that the $M^{(1)}$ corresponds to the true thresholdless process whose origin is related to momentum scattering by heterobarriers. The matrix element $M^{(2)}$ corresponds to a quasi-threshold process, and, at the quantum well width $a$ approaching infinity, it transforms into the conventional threshold matrix element.

$$
\begin{aligned}
M^{(1)} & \approx \frac{8 \pi e^{2}}{\kappa_{0}\left(q^{2}+k_{4}^{2}\right)\left(q^{2}+k_{3}^{2}\right)}\left(\frac{3 V_{c}+V_{v}}{4 E_{g}}-\frac{\kappa_{0}-\tilde{\kappa}_{0}}{\kappa_{0}+\tilde{\kappa}_{0}}\right) \times \\
& \times\left(\psi_{4}^{*}(a / 2) \psi_{1}(a / 2)\right)\left(\psi_{3}^{*}(a / 2) \psi_{2}(a / 2)\right)^{\prime}\left(1 \pm e^{-q a}\right) .
\end{aligned}
$$

Sign \pm in the last brackets is chosen according to parity of the production $\psi_{3}^{*}(x) \psi_{2}(x),+$ corresponds to the even production and - corresponds to the odd one. In the case $q a \gg 1$ this exponent may be omitted and the matrix element $M^{(1)}$ corresponds to the independent scattering at two heterobundaries. Note, that the matrix element $M$ equals to zero if the parities of productions $\psi_{3}^{*}(x) \psi_{2}(x)$ and $\psi_{4}^{*}(x) \psi_{1}(x)$ are different.

Let us now analyze $M^{(2)}$. The integral entering into $M^{(2)}$ is proportional to the sum 


$$
\int_{0}^{a} \psi_{4}^{*}(x) \psi_{3}^{*}(x) \psi_{1}(x) \psi_{2}(x) d x \propto \sum \pm \frac{\sin \left(k_{4}-k\right) a / 2}{k_{4}-k}
$$

where $k$ runs through eight different values $k= \pm k_{1} \pm k_{2} \pm k_{3}$. Of all terms in the sum (35) the largest is that for which $k=k_{1}+k_{2}+k_{3}$. 目 The contributions to the sum from other terms are less significant and will be neglected for the sake of simplicity. Then the expression for the matrix elements of the quasi-threshold Auger process takes the following form:

$$
\begin{aligned}
M^{(2)} & \approx \frac{\pi e^{2}}{\kappa_{0}\left(q^{2}+k_{4}^{2}\right)} e^{i \delta} \frac{\hbar \gamma}{E_{g}} \frac{1+2 / 3 \alpha}{1+\alpha} A_{c} A_{f} A_{c} A_{h} \frac{\sin \left(k_{f}-k_{c 1}-k_{c 2}-k_{h}\right) a / 2}{k_{f}-k_{c 1}-k_{c 2}-k_{h}} \times \\
& \times\left\{\begin{array}{cl}
q_{h} k_{c} e^{i \phi_{2,3}}+q_{c} k_{h}, & \nu_{c}= \pm \nu_{h}, \\
q_{c} q_{h} \sin \phi_{2,3}, & \nu_{c}=\mp \nu_{h} .
\end{array}\right.
\end{aligned}
$$

Here $\delta$ is an insignificant phase coefficient, $A_{i}$ denotes the normalizing constant, $\nu_{c}$ and $\nu_{h}$ are the spin indexes introduced according to (24), $\phi_{2,3}$ is the angle between lateral momenta of the electron and the hole. Note, that the matrix element for transition into continuous spectrum $(M)$ was split to $M^{(1)}$ and $M^{(2)}$ in different way than in 26 , in the purpose to make the corresponding expressions more clear.

\section{Calculation of the matrix element of Auger recombination for a transition of an excited particle into the discrete spectrum.}

We now turn our attention to analyzing the matrix element of an Auger transition in which the high-energy particle remains in bound state $\psi_{4}$. This case corresponds to the approximation $q_{4} \gg k_{4}$. The matrix element can be calculated similarly as $M^{(2)}$ earlier:

$$
M^{(3)} \approx \frac{4 \pi e^{2}}{\kappa_{0}\left(q^{2}+k_{4}^{2}\right)} \int_{-a / 2}^{a / 2}\left(\psi_{4}^{*} \psi_{1}\right)\left(\psi_{3}^{*} \psi_{2}\right) d x
$$

This integral can be readily calculated; however, the general formula is rather cumbersome and will not be presented here. We shall only make an estimate of $M_{3}$, valid in the case when bound carriers are in the ground quantum state. Then we have:

$$
M^{(3)} \approx \frac{1}{q^{2}+k_{4}^{2}} e^{i \delta} \frac{\hbar \gamma}{Z} A_{c} A_{f} A_{c} A_{h} a / 2 \alpha q_{c} q_{h} \sin \phi_{2,3} \quad\left(\nu_{c}=-\nu_{h}\right) .
$$

Here $\alpha$ is a coefficient of order unity, resulting from integrating the product of the envelopes of the carrier wave functions over the quantum well:

$$
\int_{0}^{a / 2} f_{1} f_{2} f_{3} f_{4} d x \approx a / 2 \alpha
$$


where $f_{i}=\cos k_{i} x, i$-numerates the initial and final states of particles taking part in the AR process. For wide quantum wells, in which particles may occupy different bound quantum states, $\alpha$ is given by (cf. (35):

$$
\alpha=\frac{1}{16} \sum_{\nu_{1}, \nu_{2}, \nu_{3}, \nu_{4}=0,1}(-1)^{\nu_{i} \sigma_{i}} \frac{\sin \left((-1)^{\nu_{i}} k_{i}\right) a / 2}{(-1)^{\nu_{i}} k_{i} a / 2}
$$

Here by the index $i$ is meant summation from 1 to 4 , and $\sigma_{i}$ characterizes the parity of the function $f_{i}\left(\sigma_{i}=1\right.$ and 0 for odd and even functions, respectively).

\section{AUGER RECOMBINATION COEFFICIENT}

To calculate the rate of AR, the probabilities of Auger transition per unit time (26) should be summed over all initial and final states of carriers with appropriate weights-occupation numbers.

$$
G=\frac{2 \pi}{\hbar} \sum_{\mathbf{k}_{1}, \mathbf{k}_{2}, \mathbf{k}_{3}, \mathbf{k}_{4}}\left\langle M^{2}\right\rangle \cdot f_{1} f_{2}\left(1-f_{3}\right)\left(1-f_{4}\right) \delta\left(E_{3}+E_{4}-E_{1}-E_{2}\right) .
$$

Here $f_{1}$ and $f_{2}$ are the occupancies of the initial states and $f_{3}$ and $f_{4}$ are those of final states,

$$
\left\langle M^{2}\right\rangle=\sum_{\nu_{1}, \nu_{2}, \nu_{3}, \nu_{4}}\left|M_{f i}\right|^{2}
$$

is the squared Auger matrix element, summarized over spins of initial and final states. It is more convenient to choose electrons and holes as carriers for the CHCC and CHHS processes, respectively. For high-excited states the distribution function $f_{4}$ may be set zero. Note that instead of $1-f_{3}$, we may write $\widetilde{f_{3}}$, where $\widetilde{f_{3}}$ is the distribution function for carriers of the opposite sign: holes for the CHCC process and electrons for the CHHS process.

Contributions to the rate of Auger recombination from the matrix elements $M^{(1)}, M^{(2)}$ and $M^{(3)}$ can be separated since the excited particles occupy different quantum states. The matrix elements $M^{(1)}$ and $M^{(2)}$, on the one hand, and $M^{(3)}$, on the other, describe transitions in which the excited particle occupies a state of continuous and discrete spectrum respectively. It is more difficult to separate the contributions from $M^{(1)}$ and $M^{(2)}$. Even though the physical difference between these terms is preserved, there exists a term of interference between them. At small quantum well widths the interference is essential, since both processes are in fact thresholdless; however, even when the interference is neglected we still obtain a result of correct order, reflecting all the main specific features of the AR coefficient as a function of temperature and parameters of a structure with a quantum well. For a sufficiently wide quantum well the interference between $M^{(1)}$ and $M^{(2)}$ may be neglected. Indeed, while $M^{(1)}$ as a function of quasi-momentum shows no extremum, the modulus of $M^{(2)}$ exhibits a maximum at the point 
$k_{4}(q)+k_{3}=k_{1}+k_{2}$. When the quantum well width tends to infinity, the maximum at this point is of the form of a $\delta$-function. In accordance with the aforesaid, the AR probability for wide quantum wells, corresponding to the matrix element $M^{(2)}$, has a maximum (as a function of the longitudinal momentum of the heavy hole $q_{h}$ ) at higher $q_{h}$ values than the probability associated with $M^{(1)}$. With decreasing quantum well width the maxima of these probabilities approach each other, and the region of overlapping between these matrix elements becomes larger.

The probabilities of an AR transition within the $\mathrm{CHCC}$ process, corresponding to the matrix elements $M^{(1)}$ and $M^{(2)}$, are shown in Fig. 1 as functions of the longitudinal momentum of the heavy hole at different quantum well widths. It can be seen that the interference between the thresholdless process represented by $M^{(1)}$ and the quasi-threshold process represented by $M^{(2)}$ is observed, in accordance with the aforesaid, only for narrow quantum wells. It should be noted that the AR probabilities are rather smooth functions of the longitudinal momentum of heavy hole $(q)$, since, in calculating them, summation is taken over discrete quantum states of carriers. At $q$ close to the maximum value determined by the conservation of longitudinal momentum and energy, the AR probability shows a square-root divergence eliminated upon integration with respect to $q$, i.e., in calculating the rate of AR. The probability of Auger transition for the CHHS process has a form similar to that for the CHCC process.

In line with the aforesaid, let us present the rate of AR as follows

$$
G=G_{1}+G_{2}+G_{3}
$$

where the rate $G_{1}$ corresponds to a thresholdless Auger process with the matrix element $M_{1}$, rate $G_{2}$ to a quasi-threshold Auger process with the matrix element $M_{2}$, and rate $G_{3}$ to a threshold Auger process with the matrix element $M_{3}$.

The expressions for the rates $G_{1}$ and $G_{2}$ can be derived from (41) by changing in it summation over $k_{4}$ by integration and passing from $\delta$-function with respect to energy to $\delta$-function with respect to momentum. In what follows we shall study the AR coefficients $C$ related to the rate $G$ by

$$
G=C n^{2} p \text { and } G=C p^{2} n
$$

for the CHCC and CHHS Auger processes respectively. Here $n$ and $p$ are the 2D densities of electrons and holes, respectively. For the CHCC process we have:

$C_{1} \approx \frac{32 \pi^{2} e^{4}}{\kappa_{0}^{2}} \frac{\hbar \gamma^{2}}{E_{g}^{3}} \frac{F\left(\Delta_{s o} / E_{g}\right)}{a\left(a+2 / \kappa_{c}\right)^{2}} \frac{k_{c}^{2} \kappa_{c}^{2}}{\left(k_{c}^{2}+\kappa_{c}^{2}\right)^{2}} \frac{V_{c}}{E_{g}}\left(\frac{3 V_{c}+V_{v}}{4 E_{g}}-\frac{\kappa_{0}-\tilde{\kappa}_{0}}{\kappa_{0}+\tilde{\kappa}_{0}}\right)^{2}\left\langle\frac{q_{h}^{2} k_{h}^{2}}{\left(q_{h}^{2}+k_{h}^{2}\right)^{3}} \frac{1}{k_{4}\left(q_{h}\right)}\right\rangle$,

where 


$$
F(x)=\left(\frac{1+2 x / 3}{1+x}\right)^{2} \frac{1+7 x / 9+x^{2} / 6}{(1+x / 2)(1+4 x / 9)} \text { is a coefficient of order unity, }
$$

Note, that if Kane's parameter $\gamma$ is discontinuous than the term $\frac{E_{0 c}}{2 E_{g}}\left(\frac{\delta \gamma}{\gamma}\right)^{2}$ should be added to $\left(\frac{3 V_{c}+V_{v}}{4 E_{g}}-\frac{\kappa_{0}-\tilde{\kappa}_{0}}{\kappa_{0}+\tilde{\kappa}_{0}}\right)^{2}$, where $E_{0 c}$ is the electron size quantization energy and $\delta \gamma=\gamma-\tilde{\gamma}$ is the difference between Kane's parameters in the quantum well and barrier region. However, this addition is usually negligible. The angular brackets in (43) and later denote averaging over the heavy hole distribution function. In the case of Boltzmann distribution, which is commonly the case for holes, this averaging looks like:

$$
\begin{aligned}
\left\langle f\left(q_{h}, k_{h}\right)\right\rangle & =\frac{1}{Z} \sum_{n} \int_{0}^{\infty} q_{h} f\left(q_{h}, k_{h n}\right) e^{-\frac{k_{h n}^{2}+q_{h}^{2}}{q_{T}^{2}}}, \text { where } \\
Z & =\frac{2}{q_{T}^{2}} \sum_{n} e^{-\frac{k_{h n}^{2}}{q_{T}^{2}}},
\end{aligned}
$$

$q_{T}=\sqrt{2 m_{h} T} / \hbar$ is the thermal wave vector of heavy holes, $k_{h n}$ is the wave vector corresponding to the n-th size quantization level of heavy holes.

For the CHHS process the following expression for $C_{1}$ can be derived:

$$
\begin{gathered}
C_{1} \approx \frac{2 \pi^{2} e^{4}}{\kappa_{0}^{2} \hbar} \frac{V_{c}}{E_{g}} \frac{k_{c}^{2} \kappa_{c}^{2}}{\left(k_{c}^{2}+\kappa_{c}^{2}\right)^{2}} \frac{\tilde{F}\left(\Delta_{s o} / E_{g}\right)}{a^{2}\left(a+2 / \kappa_{c}\right)} \frac{\hbar^{3}}{m_{s o}^{3}\left(E_{g}-\Delta_{s o}\right)^{3}}\left\langle\frac{k_{h 1}^{2} k_{h 2}^{2} q_{h 1}^{2}\left(q_{h 1}^{2}+q_{h 2}^{2}\right)}{\left(q_{h 1}^{2}+k_{h 1}^{2}\right)^{3}\left(q_{h 2}^{2}+k_{h 2}^{2}\right)}\right\rangle \text {, where } \\
\tilde{F}(x)=\frac{\left(2 x+3(1-x)\left(1-m_{s o} / m_{h}\right)\right)^{2}}{2 x^{2}+\left(x+3(1-x)\left(1-m_{s o} / m_{h}\right)\right)^{2}} \frac{1+2 x / 3}{1+x} .
\end{gathered}
$$

In the letter case averaging over distribution functions of two holes occurs. In deriving (44) we assumed that $E_{g}-\Delta_{s o} \gg m_{h} / m_{s o} T$.

Similarly, we obtain $C_{2}$ for the CHCC process:

$$
C_{2} \approx \frac{\pi^{2} e^{4}}{\kappa_{0}^{2}} \frac{\hbar^{3} \gamma^{4}}{E_{g}^{5}} \frac{F\left(\Delta_{s o} / E_{g}\right)}{a\left(a+2 / \kappa_{c}\right)^{2}}\left\langle\frac{q_{c}^{2} k_{h}^{2}+q_{h}^{2}\left(k_{c}^{2}+\frac{1}{2} q_{c}^{2}\right)}{\left(q_{h}^{2}+k_{h}^{2}\right) k_{4}\left(q_{h}\right)} \frac{1-\cos \left(k_{f}-k_{h}-2 k_{c}\right) a}{2\left(k_{f}-k_{h}-2 k_{c}\right)^{2}}\right\rangle .
$$

Direct calculation of the Auger coefficient $C_{2}$ for the CHHS process gives a cumbersome result. We present here a simplified expression valid for sufficiently narrow quantum wells at $k_{c} \gg q_{c}$.

$$
\begin{aligned}
C_{2} & \approx \frac{\pi^{2} e^{4}}{4 \kappa_{0}^{2}} \frac{E_{c}}{E_{g}} \frac{\hbar^{3}}{m_{s o}^{2}\left(E_{g}-\Delta\right)^{3}} \frac{\tilde{F}\left(\Delta_{s o} / E_{g}\right)}{a^{2}\left(a+2 / \kappa_{c}\right)}\left\langle\frac{1-\cos \left(k_{s o}-k_{h 1}-k_{h 2}-k_{c}\right) a}{2\left(k_{s o}-k_{h 1}-k_{h 2}-k_{c}\right)^{2}} \times\right. \\
& \left.\times \frac{q_{h 2}^{2}\left(\left(k_{s o}^{2}+k_{h 1}^{2}\right) q_{h 1}^{2}+q_{h 2}^{2} k_{h 1}^{2}+2 k_{h 1}^{2}\left(\mathbf{q}_{h 1} \mathbf{q}_{h 2}\right)+\left[\mathbf{q}_{h 1} \times \mathbf{q}_{h 2}\right]^{2}\right)}{\left(q_{h 1}^{2}+k_{h 1}^{2}\right)\left(q_{h 2}^{2}+k_{h 2}^{2}\right) k_{s o}}\right\rangle
\end{aligned}
$$

And, finally, we have for $C_{3}$ for the CHCC process:

$$
C_{3} \approx \frac{32 \pi^{2} e^{4}}{\kappa_{0}^{2} \hbar E_{g}} \frac{a}{\left(a+1 / \kappa_{c}\right)^{3}} \frac{1+\frac{7}{9} x+\frac{1}{6} x^{2}}{(1+x / 3)^{2}} \frac{1+\frac{2}{3} x}{1+x} \cdot\left\langle\frac{q_{t h}^{2}}{q_{T}^{2}} \frac{q_{c}^{2}}{\left(q_{t h}^{2}+k_{h}^{2}\right)^{3}} e^{-\frac{q_{t h}^{2}}{q_{T}^{2}}} \alpha^{2}\right\rangle_{n}
$$


Here $x=\Delta_{s o} / E_{g}, \alpha$ is a multiplier introduced in (38). In the last case we average only over discrete quantum states of heavy holes. The threshold momentum $q_{t h}$ is found from the conservation law for the energy and the longitudinal component of momentum:

$$
E_{f}\left(\sqrt{k_{f}^{2}+q_{t h}^{2}}\right)=E_{g}+\frac{\hbar^{2}\left(q_{t h}^{2}+k_{h}^{2}\right)}{2 m_{h}}+\frac{\hbar^{2}\left(k_{c 1}^{2}+k_{c 2}^{2}\right)}{2 m_{c}} .
$$

For simlicity we neglected here by longitudinal momenta of electrons, because they are small. However, we took into account the size quantization energies of electrons as they change the effective band gap in the quantum well. Expanding the energy of excited electron $E_{f}$ into a series in terms of momenta in the vicinity of $q_{t h}=Q$, where $Q$ is the electron momentum corresponding to an energy equal to $E_{g}\left(Q \approx \sqrt{\frac{4 m_{c} E_{g}}{\hbar^{2}}}\right)$, we get the following estimation for the threshold momentum:

$$
q_{t h} \approx \sqrt{\frac{4 m_{c} E_{g}}{\hbar^{2}}+\frac{3}{2}\left(k_{c}^{2}+\frac{m_{c}}{m_{h}} k_{h}^{2}\right)} .
$$

If the quantum well width tends to infinity, the threshold momentum approaches its bulk value日. Account must be taken of the fact that for wide quantum wells with large number of levels the introduced multiplier $\alpha$ (see eq. (38)) tends to a $\delta$-function expressing the conservation law for the transverse quasi-momentum component:

$$
\alpha^{2} \longrightarrow \frac{\pi}{128} a \sum \delta\left(k_{h} \pm k_{c 1} \pm k_{c 2} \pm k_{c 4}\right) .
$$

For large quantum well widths, provided that the condition $V_{c} \ll E_{g}$ holds, the inequality $C_{3} \ll C_{2}$ is valid, since the ratio $C_{3} / C_{2} \approx \sqrt{V_{c} / E_{g}}$. Hence, for wide wells $C_{3}$ may be neglected as compared with $C_{2}$. If $V_{c} \lesssim E_{g}$, the following relation holds for wide quantum wells $C_{3} / C_{2} \approx$ $\sqrt{V_{c} /\left(E_{g}-V_{c}\right)} \geq 1$. For narrow quantum wells the threshold energy of the CHCC process increases (see eq. (48)) and the AR coefficient (47) decreases relative to the bulk value by a factor

$$
e^{\frac{3 k_{c}^{2}}{2 q_{T}^{2}}} \approx e^{\frac{3 m_{c}}{2 m_{h}} \frac{E_{0 c}}{T}}
$$

The characteristic width of a quantum well for which this phenomenon becomes essential can be readily evaluated from the condition that the exponent is unity:

$$
E_{0 c} \approx T \frac{2 m_{h}}{3 m_{c}} \Leftrightarrow a \approx \pi \frac{1}{q_{T}} .
$$

Thus, at quantum well widths $a$ less than several reciprocal thermal momenta $a \lesssim \pi q_{T}$ the threshold energy $E_{t h}^{3}(a)$ becomes much higher than the bulk value $E_{t h}^{\text {bulk }}$ (see Fig. 2). For semiconductor compounds $\mathrm{A}_{I I} \mathrm{~B}_{V}$ the equality (49) is fulfilled at room temperature at a quantum well width of order of $100 \AA$. 
For the threshold CHHS process the heavy hole momenta are not specified by the threshold conditions and, therefore, we have to perform integration with respect to them. It seems impossible to derive analytically the exact result for the Auger coefficient $C_{3}$ for the CHHS process in view of the fact that the matrix element $M_{3}$ is rather cumbersome. However, an approximate expression can readily be obtained by factoring out the averaged squared matrix element from the integrand sign:

$$
\begin{aligned}
C_{3} & \approx \frac{2 \pi}{\hbar}\left\langle M_{3}^{2}\right\rangle \frac{1}{2 \pi^{2} q_{T}^{4}} \int q_{h 1} d q_{h 1} q_{h 2} d q_{h 2} d \phi_{h 1} d \phi_{h 2} e^{-\frac{q_{h 1}^{2}+q_{h 2}^{2}}{q_{T}^{2}}} \times \\
& \times \delta\left(\tilde{E}_{g}-\Delta-\frac{\hbar^{2}\left(\mathbf{q}_{\mathbf{h} \mathbf{1}}+\mathbf{q}_{\mathbf{h} \mathbf{2}}\right)^{2}}{2 m_{s o}}+\frac{\hbar^{2} q_{h 1}^{2}}{2 m_{h}}+\frac{\hbar^{2} q_{h 2}^{2}}{2 m_{h}}\right),
\end{aligned}
$$

Here $\tilde{E}_{g}=E_{g}+E_{0 c}+2 E_{0 h}-E_{0 s o}$, where $E_{0 c}, E_{0 h} E_{0 \text { so }}$ are the energies of size quantization for electrons, holes, and SO holes, respectively. Let us introduce a threshold momentum, setting it equal to

$$
Q_{t h}^{2}=\frac{2\left(\tilde{E}_{g}-\Delta\right) m_{s o}}{\hbar^{2}\left(2-\mu_{s o}\right)}
$$

where $\mu_{s o}=m_{s o} / m_{h}$. Then the expression for $C_{3}$ takes the form

$$
C_{3} \approx \frac{2 m_{s o}}{\hbar^{3} Q_{t h}^{2}} e^{-\frac{Q_{t h}^{2}}{q_{T}^{2}}}\left\langle M_{3}^{2}\right\rangle
$$

Taking into account that $Q_{t h} \gg k_{s o}$, we obtain:

$$
C_{3} \approx \frac{256 m_{s o} \pi^{2} e^{4}}{\hbar^{3} \kappa_{0}^{2}} \frac{Q_{t h}^{2}}{\left(Q_{t h}^{2}+2 k_{h}^{2}\right)^{4}} \frac{V_{c}}{E_{g}} \frac{k_{c}^{2}}{k_{c}^{2}+\kappa_{c}^{2}} \frac{\left(1-\lambda_{s o}\right)^{2}}{1+2 \lambda_{s o}^{2}} \tilde{\alpha}^{2} e^{-\frac{Q_{t h}^{2}}{q_{T}^{2}}},
$$

where $\tilde{\alpha}$ is a multiplier defined similarly as for CHCC process (see eq. (38)), $\lambda_{\text {so }}$ is derived from $\lambda_{l}$ (see eq. (A3)) by substitution $k_{s o}$ instead of $k_{l}$. Let us consider in more detail the Auger recombination coefficient $C_{2}$ for the quasi-threshold CHCC process. At $a \longrightarrow \infty$ a substitution can be made in the function being averaged in (45):

$$
\frac{1-\cos \left(k_{f}-k_{h}-2 k_{c}\right) a}{2\left(k_{f}-k_{h}-2 k_{c}\right)^{2}} \longrightarrow \frac{\pi a}{2} \delta\left(k_{f}-k_{h}-2 k_{c}\right) .
$$

This formula clearly shows the occurrence of a threshold in this limit, and the coefficient $C_{2}$ transforms into a 3D expression on being multiplied by $a^{2}$. For comparison we present both the result of Gelmont $\mathrm{B}$ for $C_{3 D}$ and our limiting expression.

$$
\begin{gathered}
C_{3 D}=6 \sqrt{2 \pi^{5}} \frac{e^{4} m_{c} \hbar^{3}}{\kappa_{0}^{2}} \frac{1}{E_{g}^{5 / 2} T^{1 / 2} m_{c}^{1 / 2} m_{h}^{3 / 2}} e^{-\frac{2 m_{c}}{m_{h}} \frac{E_{g}}{T}}, \\
C_{2} \cdot a^{2}=6 \frac{16 \sqrt{2 \pi^{5}}}{27} \frac{e^{4} m_{c} \hbar^{3}}{\kappa_{0}^{2}} \frac{1}{E_{g}^{5 / 2} T^{1 / 2} m_{c}^{1 / 2} m_{h}^{3 / 2}} e^{-\frac{2 m_{c}}{m_{h}} \frac{E_{g}}{T}} .
\end{gathered}
$$


The factor 4 in (55) results from the necessity to take into account, in calculating $M_{2}$ in accordance with (36), not only the terms with $k=k_{c 1}+k_{c_{2}}+k_{h}$, but also those with $k=$ $k_{c 1}-k_{c_{2}}+k_{h}, k=-k_{c 1}+k_{c 2}+k_{h}$, and $k=-k_{c 1}-k_{c_{2}}+k_{h}$. When the quantum well width tends to infinity, all the four terms make equal contributions to $C_{2}$. As can be seen, the only difference between the expressions (54) and (55) is in a numerical coefficient. A small discrepancy of $\approx 2 / 3$ times is due to the necessity to distinguish between the size quantization momenta of the two localized electrons: $k_{c 1} \neq k_{c 2}$, besides the expression (54) was derived when $\Delta_{s o}$ tends to infinity, while obtaining the expression (55) it was assumed that $\Delta_{s o} \lesssim E_{g}$. In calculating (55) we neglected the quantity $V_{c}$ as compared with $E_{g}$. In the general case, $\left(C_{2}+C_{3}\right) a^{2}$ should be written instead of $C_{2} a^{2}$ to make expression (55) valid. However, the limiting transition from the quasi-threshold to the threshold Auger process (see eq. (53)) can be realized only for very large quantum wells. Analyzing the probability of Auger transition as a function of the heavy hole momentum, one can obtain a qualitative criterion for this transition. As mentioned above, the quasi-threshold Auger process dominates in wide quantum wells. The probability of this process has two characteristic extrema (see eq. (45])). The first of them corresponds to the maximum of squared Auger transition matrix element in the vicinity of the threshold momentum. The width of this maximum is of the order of the inverse quantum well width. The second extremum lies in the vicinity of the thermal momentum of heavy holes. The Auger coefficient $C_{2}$ can be estimated as a sum of the probabilities taken in these extrema, multiplied by the corresponding widths. Then

$$
C_{2} \approx C_{2}^{t h}\left(Q_{h} \approx q_{t h}\right)+C_{2}^{T}\left(Q_{h} \approx q_{T}\right),
$$

where $Q_{h}$ is the value of the heavy hole momentum: $Q_{h}^{2}=k_{h}^{2}+q_{h}^{2}$;

$$
\frac{C_{2}^{T}}{C_{2}^{t h}} \approx \frac{\lambda_{E_{g}}}{a}\left(\frac{T}{E_{t h}}\right)^{3 / 2} e^{\frac{E_{t h}}{T}} .
$$

Here $\lambda_{E_{g}} \approx 2 \pi / q_{t h}$ is a characteristic wave length of an electron having energy close to $E_{g}$. Comparing the terms $C_{2}^{t h}$ and $C_{2}^{T}$ one can obtain the criterion of the transition from the quasithreshold to the threshold Auger process:

$$
a \gg a_{c}, \text { wherea } a_{c}=\lambda_{E_{g}}\left(\frac{T}{E_{t h}}\right)^{3 / 2} e^{\frac{E_{t h}}{T}} .
$$

For semiconductors with an energy gap of the order of $1 \mathrm{eV}$ the critical width $\left(a_{c}\right)$ may be as large as several thousand Angstroms. However, the value $a_{c}$ is considerably larger than the free path length of carriers in semiconductors. This obviously shows that the correct derivation of Auger rate in homogeneous semiconductors should involve momentum scattering process if the critical width $a_{c}$ there exceeds the free path length 25 . 
With decreasing quantum well width, the maximum of the probability $w_{2}$ as a function of the heavy hole momentum shifts to lower values (see Fig. 1). This reduces the threshold energy of the process, and, as a consequence, makes weaker the temperature dependence of the AR coefficient.

Figure 2 shows the threshold energy of the CHCC process as a function of quantum well width for all the three mechanisms of Auger recombination $C_{1}, C_{2}$, and $C_{3}$ separately and for the overall process $C=C_{1}+C_{2}+C_{3}$, found from the formula:

$$
E_{t h}^{i}(T)=T^{2} \frac{d \ln C_{i}}{d T}, \quad i=1,2,3
$$

The threshold energy for the quasi-threshold Auger process (see Fig. 2) is less than its bulk value. The reason is that the critical quantum well width $a_{c} \approx 2000 \stackrel{\circ}{A}$ is greater than the maximum width shown in the figure. The threshold energy for the thresholdless Auger process decreases with quantum well width and becomes negative at a certain thickness. This is due to the fact that the Auger coefficient $C_{1}$ decreases with increasing temperature for wide enough quantum wells (see Fig. 5). With increasing quantum well width, the total threshold energy tends to its limiting value $E_{t h}^{b u l k}$ denoted in the figure.

We now turn our attention to the thresholdless Auger process. As already noted, the probability of a thresholdless Auger transition shows no extrema as a function of the heavy hole momentum. Therefore, the coefficient $C_{1}$ has a weak nonexponential temperature dependence. This phenomenon was first studied by Zegrya and Kharchenkol. In addition, the function $C_{1}(T)$ is nonmonotonic and has a maximum. The presence of this maximum can readily be explained. At low temperature and, correspondingly, small longitudinal momenta of carriers their wave functions are nearly orthogonal and the $C_{1}$ value is small. With increasing temperature, the characteristic momentum transferred in Coulomb interaction (approximately equal to the thermal momentum of heavy hole) grows. This is the reason why at low temperature the Auger coefficient increases with temperature. As the temperature is elevated further, the AR coefficient $C_{1}$ passes through a maximum and starts to decrease, since the long-range Coulomb interaction responsible for the Auger process is low for large transferred momenta. The temperature at which the maximum occurs can readily be evaluated by equating the energy of size quantization of holes to temperature: $T \approx \frac{\hbar^{2} \pi^{2}}{2 m_{h} a^{2}}$. Note that there would be no such maximum if the overlap integral $I_{c h}$ were taken to be proportional to the momentum transferred. Such an assumption, having in our opinion no justification for the majority of structures investigated, is frequently used (see, e.g., ref.17) and gives incorrect expressions for the rate of AR and incorrect dependence of this quantity on temperature and quantum well parameters.

The AR coefficient $C_{1}$ depends rather strongly on the quantum well width $a$. Depending on which term predominates in (43), the coefficient $C_{1}$ decreases with increasing $a$ either as $1 / a^{3}$ or as $1 / a^{5}$ or as $1 / a^{7}$. In any case, even being multiplied by $a^{2}, C_{1}$ remains a decreasing 
function of quantum well width. For this reason such a process may be dominant only for sufficiently narrow quantum wells. At $a \approx 1 / \kappa_{c}$ the coefficient $C_{1}$ exhibits a maximum related to the weak overlapping of carrier wave functions. With the quantum well width decreasing further, the rate of the thresholdless Auger process falls gradually. The similar expression for $C_{1}$ in the CHCC process was obtained by Dyakonov and Kachorovskii, and Zegrya et al. 4. . 1 .

Figure 3 shows the AR coefficients $C_{1}$ and $C_{2}$ for the CHCC process as functions of quantum well width at different temperatures for a model structure based on InGaAsP. It can be seen that, firstly, both $C_{1}$ and $C_{2}$ show a sharply pronounced maximum. Interestingly, the positions of these maxima are practically temperature-independent. Secondly, the relative role of the quasi-threshold process becomes more important with increasing temperature. The threshold process is not shown in the figure, since at the quantum well widths considered the coefficient $C_{3}$ is much smaller (by several orders of magnitude) than $C_{1}$ and $C_{2}$. For this reason the dependence of $C_{3}$ on quantum well width is shown separately in Fig. 4. Note that for this process the maximum is achieved at a much wider quantum well than for the quasi-threshold or thresholdless process. This is in the first place due to the reduction of the threshold energy of the threshold process with increasing quantum well width (Fig. 2), rather than to the overlapping of the wave functions. The coefficients $C_{1}$ and $C_{2}$ as functions of quantum well width for the CHHS process qualitatively agree with the curves presented in Fig. 3.

Figure 5 shows the temperature dependence of the overall AR coefficient for the CHCC process and the partial contributions from the thresholdless and quasi-threshold mechanisms at different quantum well widths. It can be seen that at low temperature and sufficiently wide quantum wells the thresholdless Auger process predominates $\left(C_{1}>C_{2}\right)$, and at high temperature, conversely, the quasi-threshold process becomes more important $\left(C_{2}>C_{1}\right)$. Therefore, the curve describing the temperature dependence of the overall AR coefficient has a characteristic shape with a maximum and a minimum. With increasing quantum well width, both the maximum and the minimum of the AR coefficient shift to lower temperature and, in the limit of infinitely wide quantum well, disappear. Thus, in the case of a homogeneous semiconductor the AR coefficient is a monotonic function of temperature. Note that the Boltzmann distribution of carriers was used in calculating the Auger coefficients as functions of temperature. At low temperature both electrons and holes are as a rule described by Fermi-Dirac distribution function. Thus, the average momenta of particles participating in the Auger transition depend on temperature only slightly. As a result, at low temperatures the Auger coefficient is a smoother function of temperature than in the case of the Boltzmann statistic and it does not tend to zero at $T \rightarrow 0$. Figure 6 shows the thresholdless Auger coefficient $C_{1}$ versus temperature at various Fermi energies for quantum wells with different widths. Essential discrepancies between the values of the Auger coefficients for the Fermi-Dirac and Boltzmann distributions take place only 
in the case $T \ll E_{F}$, where $E_{F}$ is the Fermi energy for holes. This condition can be realized only at very low temperature where the Auger process ceases to be an important mechanism of recombination.

\section{PHONON-ASSISTED AUGER RECOMBINATION IN QUANTUM WELLS}

At high temperature the threshold AR process predominates in the homogeneous semiconductor

$\left(C_{3 D} \propto e^{-\frac{E_{t h}}{T}}\right)$. However, at sufficiently low temperature such a process becomes exponentially weak. In this case the rate of the Auger process is no longer determined by the scattering of two free electrons. Mechanisms leading to threshold removal are to be taken into account. It is commonly believed that the primary mechanism of this kind is emission or absorption of a virtual optical phonon. At the expense of a large momentum transferred to the phonon, the AR threshold for heavy holes is removed, and the rate of such an Auger process is a power-law function of temperature盷, 27. The probability of the phonon-assisted AR is calculated in terms of the second-order perturbation theory in electron-electron and electron- phonon interaction 24. However, the possibility of removing the threshold through interaction with phonons is not the only one. At high hole concentrations, hole-hole scattering may become a more effective mechanism of removing the threshold for the Auger process. This is why the question of the AR mechanism in homogeneous semiconductors at low temperature still remains open. We shall discuss this problem in more detail elsewhere 25 . In the present work we follow the commonly accepted viewpoint that there exists a competition between the phonon-assisted and direct AR processes. For quantum wells the situation differs strongly from the $3 \mathrm{D}$ case, owing to the presence of a thresholdless process even in the first order of the perturbation theory. Therefore, it is a priori evident that the conditions under which the phonon-assisted AR dominates over the direct process depend strongly on quantum well width.

As already noted, in the 2D case there exist three AR processes: threshold, quasi-threshold, and thresholdless. It will be recalled that in sufficiently narrow quantum wells thresholdless and quasi-threshold AR processes are predominant at all temperatures. Below, it will be shown that the electron-phonon interaction may affect significantly the rate of AR at low temperature. The AR coefficient for a phonon-assisted CHCC process with a threshold matrix element of electron-electron interaction is comparatively easily calculated when 27

$$
E_{g} \gg 2 \mu E_{g} \gg \hbar \omega_{L O}, T
$$

where $\omega_{L O}$ is the optical phonon frequency, $\mu=m_{c} / m_{h}$. It can be shown that the coefficient of phonon-assisted AR is related to the previously calculated AR coefficient (55) by

$$
C_{p h}^{3} \approx C_{3} \frac{e^{2} \hbar \omega_{L O}}{2 \bar{\kappa} a} \frac{T}{E_{t h}^{2 D}} g\left(a, k_{t h}\right) \frac{1}{e^{\frac{\hbar \omega_{L O}}{T}}-1}\left[\frac{e^{\frac{\hbar \omega_{L O}}{T}}}{\left(E_{t h}^{2 D}-\hbar \omega_{L O}\right)^{2}}+\frac{1}{\left(E_{t h}^{2 D}+\hbar \omega_{L O}\right)^{2}}\right] e^{\frac{E_{t h}^{2 D}}{T}}
$$


where $\bar{\kappa}=\frac{\kappa_{0} \kappa_{\infty}}{\kappa_{0}-\kappa_{\infty}}, \kappa_{\infty}$ is the high-frequency permittivity of the medium, $g\left(a, k_{t h}\right)$ is a factor reflecting the $2 \mathrm{D}$ character of holes 28

$$
g\left(a, k_{t h}\right)=k_{t h}^{2} a^{2}\left[\frac{1}{2 k_{t h}^{2} a^{2}}+\frac{1}{4\left(\pi^{2}+k_{t h}^{2} a^{2}\right)}\right]\left[1-\frac{1-e^{-2 k_{t h} a}}{2 k_{t h} a} \frac{2 \pi^{4}}{\left(\pi^{2}+k_{t h}^{2} a^{2}\right)\left(2 \pi^{2}+3 k_{t h}^{2} a^{2}\right)}\right] .
$$

For comparison, we present an AR coefficient calculated for the phonon-assisted 3D case

$$
C_{p h}^{3 D} \approx C^{3 D} \frac{e^{2} \hbar \omega_{L O}}{2 \sqrt{\pi} \bar{\kappa}}\left(\frac{T}{E_{t h}^{3 D}}\right)^{3 / 2} \frac{k_{t h}}{e^{\frac{\hbar \omega_{L O}}{T}}-1}\left[\frac{e^{\frac{\hbar \omega_{L O}}{T}}}{\left(E_{t h}^{3 D}-\hbar \omega_{L O}\right)^{2}}+\frac{1}{\left(E_{t h}^{3 D}+\hbar \omega_{L O}\right)^{2}}\right] e^{\frac{E_{t h}^{3 D}}{T}} .
$$

It can be seen that the results for $2 \mathrm{D}$ and $3 \mathrm{D}$ phonon-assisted $\mathrm{AR}$ processes with threshold matrix elements of electron-electron interaction are closely allied. A significant difference for the case of narrow quantum wells is that the threshold energy $E_{t h}^{2 D}$ increases owing to the presence of carrier size quantization levels (see eq. (48)). Correspondingly, the criterion for predominance of the phonon-assisted AR process $\left(C_{3}^{p h}\right)$ over the direct threshold Auger process $\left(C_{3}\right)$ in quantum wells is met at somewhat higher temperature than in the 3D case. However, as already noted (see Section III), the rate of the threshold Auger process in narrow quantum wells is in itself by several orders of magnitude lower than those of the thresholdless and quasithreshold processes $\left(C_{3} \ll\left(C_{1}, C_{2}\right)\right)$. Hence, the phonon-assisted AR process with a threshold matrix element of electron-electron interaction cannot compete with thresholdless and quasithreshold processes either $\left(C_{p h}^{3} \ll C_{1}, C_{2}\right)$.

Let us now consider a phonon-assisted Auger process with the thresholdless matrix element $\left(M_{e e}\right)$ for the CHCC process. Direct calculation of the probability of this process results in a singularity at the energy of a hole in the virtual state energy equal to the sum of the energies of the optical phonon and the hole in the final state:

$$
w_{i \rightarrow f}= \pm \frac{2 \pi}{\hbar} \sum_{s} \frac{\left|M_{e e}\right|^{2}\left|M_{h p}\right|^{2}}{\left(E_{s} \mp \hbar \omega_{L O}-E_{h}\right)^{2}} \frac{e^{ \pm \frac{\hbar \omega_{L O}}{T}}}{e^{ \pm \frac{\hbar \omega_{L O}}{T}}-1} \delta\left(E_{i}-E_{f}\right) d \nu_{f}
$$

where $E_{s}$ is the energy of the virtual hole, $a n d M_{e p}$ is the matrix element of scattering of the virtual hole by an optical phonon, with the signs plus and minus corresponding to phonon emission and absorption, respectively. Note that in the 3D case the summation over intermediate states is in fact reduced to taking a single summand. In the $2 \mathrm{D}$ case, nothing of the kind occurs, and the sum is taken over discrete quantum states. To eliminate the divergence in the denominator of the expression (64), account must be taken of transitions not into stationary, but quasi-stationary states, i.e., states with complex energy. In this case the pole (64) will transform into a region of complex energy values:

$$
w_{i \rightarrow f} \propto \frac{1}{\left(E_{s} \mp \hbar \omega_{L O}-E_{h}\right)^{2}+\Gamma^{2}},
$$


where $\Gamma=\hbar / \tau$. The characteristic lifetimes $\tau$ corresponding to these states may vary over a wide range, depending on temperature, free carrier density, etc. It only makes sense to consider a resonant phonon-assisted process in terms of the second-order perturbation theory when the halfwidths of the quasi-stationary hole and the phonon states are less than the energy of the optical phonon $\left(\hbar \omega_{L O}\right)$. Otherwise, the Auger coefficient must be calculated in the first order of the perturbation theory, using the Lorentz function $\left(f(\Delta E)=\frac{1}{\pi} \frac{\Gamma}{\Delta E^{2}+\Gamma^{2}}\right)$ instead of the $\delta$-function expressing the energy conservation law. For a phonon-assisted AR process with a quasi-threshold matrix element of electron-electron interaction both the resonant and virtual Auger processes are possible, with the former predominant in narrow quantum wells and the latter in sufficiently wide quantum wells.

In the general case the Auger coefficient for a phonon-assisted process with the quasithreshold and the thresholdless matrix elements $M_{e e}$ may be written as

$$
C_{p h}=C_{p h}^{1}+C_{p h}^{2},
$$

where

$$
\begin{aligned}
C_{p h}^{1}= & \frac{\pi \omega e^{2}}{\bar{\kappa} Z} \frac{e^{\hbar \omega_{L O} / T}}{e^{\hbar \omega_{L O} / T}-1} \sum_{m, n, \nu_{n}} \int \frac{d^{2} Q}{(2 \pi)^{2}} \frac{d^{2} q_{h}}{(2 \pi)^{2}}\left(\frac{\partial E_{4}}{\partial k_{4}}\right)^{-1} \\
& \frac{\mid M_{e e}\left(n, \mathbf{q}_{\mathbf{h}}+\left.\mathbf{Q}\right|^{2}\right.}{\left(\frac{\hbar^{2}\left(n^{2}-m^{2}\right) \pi^{2}}{2 a^{2} m_{h}^{2}}+\frac{\hbar^{2}\left(\mathbf{q}_{\mathbf{h}}+\mathbf{Q}\right)^{2}}{2 m_{h}}-\frac{\hbar^{2} q_{h}^{2}}{2 m_{h}}-\hbar \omega_{L O}\right)^{2}+\Gamma^{2}} J_{n, m}(Q) f_{h}\left(m, q_{h}\right) \\
C_{p h}^{2}= & \frac{\pi \omega e^{2}}{\bar{\kappa} Z} \frac{1}{e^{\hbar \omega_{L O} / T}-1} \sum_{m, n, \nu_{n}} \int \frac{d^{2} Q}{(2 \pi)^{2}} \frac{d^{2} q_{h}}{(2 \pi)^{2}}\left(\frac{\partial E_{4}}{\partial k_{4}}\right)^{-1} \\
& \frac{\mid M_{e e}\left(n, \mathbf{q}_{\mathbf{h}}+\left.\mathbf{Q}\right|^{2}\right.}{\left(\frac{\hbar^{2}\left(n^{2}-m^{2}\right) \pi^{2}}{2 a^{2} m_{h}^{2}}+\frac{\hbar^{2}\left(\mathbf{q}_{\mathbf{h}}+\mathbf{Q}\right)^{2}}{2 m_{h}}-\frac{\hbar^{2} q_{h}^{2}}{2 m_{h}}+\hbar \omega_{L O}\right)^{2}+\Gamma^{2}} J_{n, m}(Q) f_{h}\left(m, q_{h}\right) .
\end{aligned}
$$

Here:

$$
\begin{gathered}
Z=\sum_{m} \int \frac{d^{2} q_{h}}{(2 \pi)^{2}} f_{h}\left(m, \mathbf{q}_{\mathbf{h}}\right), \\
J_{n, m}(Q)=\frac{a}{2} \frac{\left(1+\delta_{m, n}\right)\left((m+n)^{2} \pi^{2}+Q^{2} a^{2}\right)+(m-n)^{2} \pi^{2}+Q^{2} a^{2}}{\left((m+n)^{2} \pi^{2}+Q^{2} a^{2}\right)\left((m-n)^{2} \pi^{2}+Q^{2} a^{2}\right)}(1-\varepsilon), \\
\varepsilon=\frac{Q a\left(1-(-1)^{m+n} e^{-Q a}\right) 32 \pi^{4} n^{2} m^{2}}{\left((m+n)^{2} \pi^{2}+Q^{2} a^{2}\right)\left((m-n)^{2} \pi^{2}+Q^{2} a^{2}\right)} \times \\
\times \frac{1}{\left(1+\delta_{m, n}\right)\left((m+n)^{2} \pi^{2}+Q^{2} a^{2}\right)+(m-n)^{2} \pi^{2}+Q^{2} a^{2}} .
\end{gathered}
$$

The function $J_{n, m}(Q)$ has been calculated for a nondegenerate band. In the case of phonon scattering by heavy holes its value will be somewhat lower. However, this fact is insignificant 
for our purposes. For momenta of bound electrons in the matrix element of electron-electron interaction in (67) should be substituted their mean thermal values. The Auger coefficients $C_{p h}^{1}$ and $C_{p h}^{2}$ correspond, respectively, to phonon emission and absorption. Irrespective of the type of the matrix element of Coulomb interaction, the phonon-assisted Auger process is thresholdless. This corresponds to the main contribution to the Auger coefficient $C_{p h}$ coming from the hole momenta of the same order of magnitude as the thermal momentum. Therefore, in calculating $C_{p h}$ me may substitute for the longitudinal hole momentum $q_{h}$ its mean thermal value. This simplifies considerably the expression for the Auger coefficient:

$$
\begin{aligned}
C_{p h}^{1} \approx & \frac{\pi \omega e^{2}}{\bar{\kappa}} \frac{e^{\hbar \omega_{L O} / T}}{e^{\hbar \omega_{L O} / T}-1} \sum_{m, n, \nu_{n}} \int \frac{d^{2} Q}{(2 \pi)^{2}}\left\langle\left(\frac{\partial E_{4}}{\partial k_{4}}\right)^{-1}\right\rangle \\
& \frac{\mid M_{e e}\left(n, \mathbf{q}_{\mathbf{h}}+\left.\mathbf{Q}\right|^{2}\right.}{\left(\frac{\hbar^{2}\left(n^{2}-m^{2}\right) \pi^{2}}{2 a^{2} m_{h}^{2}}+\frac{\hbar^{2}\left(\mathbf{q}_{\mathbf{h}}+\mathbf{Q}\right)^{2}}{2 m_{h}}-\frac{\hbar^{2} q_{h}^{2}}{2 m_{h}}-\hbar \omega_{L O}\right)^{2}+\Gamma^{2}} J_{n, m}(Q) \frac{p_{m}}{\sum_{i} p_{i}}, \\
C_{p h}^{2} \approx & \frac{\pi \omega e^{2}}{\bar{\kappa}} \frac{1}{e^{\hbar \omega_{L O} / T}-1} \sum_{m, n, \nu_{n}} \int \frac{d^{2} Q}{(2 \pi)^{2}}\left\langle\left(\frac{\partial E_{4}}{\partial k_{4}}\right)^{-1}\right\rangle \\
& \frac{\mid M_{e e}\left(n, \mathbf{q}_{\mathbf{h}}+\left.\mathbf{Q}\right|^{2}\right.}{\left(\frac{\hbar^{2}\left(n^{2}-m^{2}\right) \pi^{2}}{2 a^{2} m_{h}^{2}}+\frac{\hbar^{2}\left(\mathbf{q}_{\mathbf{h}}+\mathbf{Q}\right)^{2}}{2 m_{h}}-\frac{\hbar^{2} q_{h}^{2}}{2 m_{h}}+\hbar \omega_{L O}\right)^{2}+\Gamma^{2}} J_{n, m}(Q) \frac{p_{m}}{\sum_{i} p_{i}},
\end{aligned}
$$

where

$$
p_{m}=e^{\frac{-k_{h m}^{2}}{q_{T}^{2}}}, \quad q_{h}=\frac{\sqrt{\pi}}{2} \sqrt{\frac{2 m_{h} T}{\hbar^{2}}}
$$

Expressions (67) and (68) can be analyzed easily when the temperature is much lower than the optical phonon energy. In this case the thermal momenta of holes $q_{h}$ may be neglected as compared with the phonon momentum $Q$ which is approximately equal to the momentum of virtual hole. It is readily seen that the probability of Auger transition as a function of $Q$ for phonon emission has two extrema. The first of these corresponds to the minimum of the denominator in (67) and is related to a resonant Auger transition. Note that for an Auger transition with phonon absorption no extremum of this kind is observed and there is no resonant process. The second extremum corresponds to the maximum of the squared matrix element and, as a rule, is related to a virtual Auger transition. For sufficiently wide quantum wells the matrix element of the electron-electron interaction as a function of the heavy hole momentum has a form close to the $\delta$-function. In this case the probability of the transition is the highest near the threshold momentum, and the process of scattering by phonons is virtual. With decreasing quantum well width, the $\delta$-function broadens for the quasi-threshold matrix element, and, in addition, the role of the thresholdless matrix element, only slightly depending on $Q$, becomes more significant. This enhances the resonant Auger transition and weakens the virtual process. For narrow quantum wells the matrix element of Coulomb electron-electron 
interaction depends on $Q$ only slightly, and, therefore, the resonant process is predominant. It can readily be shown that in this case the following estimation is valid for the AR coefficient of the phonon-assisted Auger process:

$$
C_{p h} \approx \frac{\omega_{L O} e^{2} m_{h} a}{8 \tilde{\kappa} \hbar \Gamma} J_{1,1}\left(Q_{0}\right) \frac{2 \pi}{\hbar} \frac{3 k\left(E_{g}\right)}{4 E_{g}}\left|M_{e e}\left(Q_{0}\right)\right|^{2},
$$

where $Q_{0}=\sqrt{\frac{2 m_{h} \omega_{L O}}{\hbar}}$. Hence immediately follows that the phonon-assisted to direct $\mathrm{AR}$ coefficient ratio has the form

$$
\frac{C_{p h}}{C} \approx \frac{\Gamma_{h p}}{\Gamma} \frac{\left(M_{e e}\left(Q_{O}\right)\right)^{2}}{\left(M_{e e}\left(q_{T}\right)\right)^{2}}
$$

where $C=C_{1}+C_{2}$ is the Auger coefficient for the direct process, $\Gamma_{h p}=\frac{\hbar}{\tau_{h p}}, \tau_{h p}$ is the time of a hole scattering by an optical phonon; $q_{T}$ is the thermal momentum of holes. It can be seen that the phonon-assisted Auger process may dominate over the direct one only in the case when the the values of $\Gamma_{p h}$ and $\Gamma$ are close to each other or at extremely low temperature when the ratio of the matrix elements taken at the momenta $Q_{O}$ and $q_{T}$ is large. Note that at high nonequilibrium carrier densities, when Auger recombination becomes at all significant, the hole-hole scattering turns out to be, as a rule, a much more effective mechanism of relaxation than the hole-phonon scattering. This results in a small ratio $\Gamma_{p h} / \Gamma$. Therefore, the direct Auger recombination dominates over the phonon-assisted process down to very low temperatures. Figure 7 shows the coefficients of a phonon-assisted Auger transition $\left(C_{p h 1}\right.$ and $\left.\left(C_{p} h 2\right)\right)$ corresponding to the thresholdless and quasi-threshold matrix elements as a function of temperature for different quantum well widths. As a halfwidth $\mathrm{G}$ is taken a characteristic value of $20 \mathrm{meV}$.

\section{DISCUSSION}

Our analysis has shown that for the CHCC and CHHS processes in semiconductor structures with quantum wells there exist three AR mechanisms: threshold, quasi-threshold, and thresholdless. The coefficients of these processes $C_{1}, C_{2}$, and $C_{3}$ depend in different ways both on temperature and quantum well parameters: the heights of heterobarriers for electrons and holes $\left(V_{c}\right.$ and $\left.V_{v}\right)$ and the well width (see Figs. $3-5$ ). In the limit $a \longrightarrow \infty$ the sum of the quasi-threshold and the threshold AR coefficients multiplied by squared quantum well width, $C_{2} a^{2}+C_{3} a^{2}$, approaches the bulk AR coefficient $C^{3 D}$, with the product $C_{1} a^{2}$ approaching zero (Fig. 8). For sufficiently narrow quantum wells the 2D AR coefficient multiplied by $a^{2}$ exceeds the $3 \mathrm{D}$ value, owing to the predominance of the thresholdless and quasi-threshold AR processes. Thus, Auger recombination in quantum wells is enhanced as compared with that in a homogeneous semiconductor. This enhancement is more pronounced at low temperature. Under these 
conditions the 3D AR coefficient $C^{3 D}$ is small because of the presence of an exponentially small multiplier (see eq. (54)). Note that the entire analysis of the AR coefficients $\left(C_{1}, C_{2}, C_{3}\right)$ as functions of temperature and quantum well parameters is qualitatively applicable to the same extent to both the CHCC and CHHS Auger processes. However, since no model structures with quantum wells have been specified, we illustrated these relations by the example of the CHCC process.

Note that the AR in quantum wells may be suppressed substantially if the following conditions are met $\left(V_{c}, V_{v}\right)>E_{g}$ and $E_{2}-E_{1}>E_{g}\left(E_{1}\right.$ and $E_{2}$ are the energies of the first and second carrier size quantization levels ) 29 ; i.e., in the case when the energy of an excited particle is insufficient for a transition into the continuous spectrum or to a next size quantization level. For these conditions to be fulfilled, a structure is to be created with deep and narrow quantum wells for both electrons and holes. The presently existing technologies can produce structures of this kind based on InAs/AlSb 30 and InAs/GaSb/AlSb 1 . In these deep quantum wells only the threshold AR mechanism, corresponding to the coefficient $C_{3}$, is operative. This coefficient may be smaller by several orders of magnitude than the Auger coefficients for the thresholdless and quasi-threshold processes $\left(C_{1}, C_{2}\right)$ in shallow quantum wells $\left(\left(V_{c}, V_{v}\right)<E_{g}\right)$.

It is also shown that the phonon-assisted AR process undergoes significant changes. Similarly to the direct AR there exist three different phonon mechanisms $\left(C_{p h}^{3}, C_{p h}^{2}, C_{p h}^{1}\right)$ corresponding to the threshold, quasi-threshold, and thresholdless matrix elements of electron-electron interaction. The first process is quite similar to its 3D counterpart. However, for narrow quantum wells this process is much weaker than the thresholdless and threshold Auger processes. It is this process with the participation of phonons that is considered in the literature to be principal AR process in quantum wells32.33. AR phonon-assisted processes with the quasi-threshold and the thresholdless matrix elements of electron-electron interaction may be resonant. At low temperature they can compete with direct AR processes. However, owing to the lack of any strong temperature dependence in the latter, such a competition is possible at much lower temperatures than in the 3D case (see Fig. 7). Thus in narrow quantum wells the direct (thresholdless) Auger recombination dominates over the phonon-assisted process in a wider interval of temperatures than in the 3D case. With increasing quantum well width, the resonant scattering by phonons becomes weaker, and we pass to the conventional 3D conditions.

It should be emphasized once again that at high densities of nonequilibrium carriers in a homogeneous semiconductor the phonon-assisted AR process may be less intensive than the Auger recombination, with the subsequent hole-hole scattering eliminating the threshold 25. 


\section{CONCLUSIONS}

The following principal results were obtained in the work

1. It is shown that three main mechanisms of Auger recombination are operative simultaneously in quantum wells:
(a) threshold
(b) quasi-threshold
(c) thresholdless

The first two pass, in the limit of infinitely wide quantum well, into 3D Auger recombination, and the coefficient of the thresholdless Auger process tends to zero.

2. It is demonstrated that in the case of narrow quantum wells the Auger coefficients of the quasi-threshold and thresholdless processes show weak power-law dependence on temperature. Their values much exceed the 3D coefficient related to the squared quantum well width. At the same time the coefficient of 2D threshold Auger recombination (a) has a higher threshold energy than in the $3 \mathrm{D}$ case $\left(E_{t h}^{2 D}>E_{t h}^{3 D}\right)$, with the corresponding AR coefficient smaller than the 3D expression divided by the squared quantum well width.

3. It is shown that the quasi-threshold Auger process predominates in wide enough quantum wells. The threshold energy of this process is an increasing function of temperature (see Fig. 2). The critical quantum well width at which the threshold energy for the quasithreshold Auger process becomes equal to the bulk value, is found. The critical width $a_{c}$ strongly (exponentially) depends on temperature. At room temperatures its value may be up to several thousand Angstroms for semiconductors with $E_{g} \approx 1 \mathrm{eV}$.

4. In narrow quantum wells the phonon-assisted AR process is resonant. At high carrier densities it is less intensive than the direct thresholdless Auger process down to very low temperature.

\section{ACKNOWLEDGMENTS}

The authors would like to thank R.A. Suris and V.I. Perel for initiating this work. The work was supported in part by the Russian Foundation for Basic Research (grant nos. 96-02-17952 and 97-02-18151), INTAS (grant no. 94-1172), and Russian State Program "Physics of Solid State Nanostructures." 


\section{APPENDIX A: WAVE FUNCTIONS OF CARRIES IN A RECTANGULAR QUANTUM WELL}

\section{Holes}

Selecting the coordinate system so that the longitudinal component of the wave vector coincide with the $y$ axis and performing a Fourier transform in this plane we obtain the following expressions for the wave functions of carriers.

Heavy holes:

$$
\begin{aligned}
\boldsymbol{\psi}_{h}(q, x) & =H_{1}\left(\begin{array}{c}
q \cos k_{h} x \xi \\
-i k_{h} \sin k_{h} x \xi \\
-k_{h} \sin k_{h} x \xi+q \cos k_{h} x \eta
\end{array}\right)+ \\
& +H_{2}\left(\begin{array}{c}
q \sin k_{h} x \eta \\
i k_{h} \cos k_{h} x \eta \\
-q \sin k_{h} x \xi-k_{h} \cos k_{h} x \eta
\end{array}\right)
\end{aligned}
$$

Where $\xi=1 / \sqrt{2}\left(\begin{array}{r}1 \\ -1\end{array}\right), \quad \eta=1 / \sqrt{2}\left(\begin{array}{l}1 \\ 1\end{array}\right), H_{1}$ and $H_{2}$ are the normalizing constants.

Light holes:

$$
\begin{gathered}
\boldsymbol{\psi}_{l}(q, x)=L_{1}\left(\begin{array}{c}
k_{l} \sin k_{l} x \eta-\lambda_{l} q \cos k_{l} x \xi \\
-i q \cos k_{l} x \eta+i \lambda_{l} k_{l} \sin k_{l} x \xi \\
-\lambda_{l} k_{l} \sin k_{l} x \xi+\lambda_{l} q \cos k_{l} x \eta
\end{array}\right)+ \\
+L_{2}\left(\begin{array}{c}
-k_{l} \cos k_{l} x \xi-\lambda_{l} q \sin k_{l} x \eta \\
-i \lambda_{l} k_{l} \cos k_{l} x \eta-i q \sin k_{l} x \xi \\
-\lambda_{l} q \sin k_{l} x \xi-\lambda_{l} k_{l} \cos k_{l} x \eta
\end{array}\right) \\
\psi_{s l}=\frac{i \hbar \gamma\left(k_{l}^{2}+q^{2}\right)}{E_{g}+\delta-E}\left[L_{1} \cos k_{l} x \eta+L_{2} \sin k_{l} x \xi\right] \\
\lambda_{l}=\frac{\delta}{E+2 \delta-\hbar^{2} k_{l}^{2} / 2 m_{h}}
\end{gathered}
$$

The wave functions of SO holes are similar to those of light holes.

A change to functions of another symmetry in the above expressions can be performed by the formal substitution $\xi \leftrightarrow \eta$ for $|s\rangle-,|x\rangle$-, and $|y\rangle$-components and $\xi \leftrightarrow-\eta$ for $|z\rangle$-components. The wave functions of carriers in the barrier region may be obatined similarly to (A1 A3). 
If wave functions of two or more particles are considered together, then in general their $z$-components of quasi-momentum cannot become zero simultaneously. A change to a function with arbitrary direction of quasi-momentum can be performed using the rotation matrix:

$$
D_{\varphi}=R_{\varphi} \otimes S_{\varphi}
$$

where $R_{\varphi}$ acts on the coordinate components of the wave function, and $S_{\varphi}$ on the spinor components. The Euler angles for a rotation in the plane $y z$ by an angle $\varphi$ are

$$
\Phi=-\pi / 2 \quad, \Theta=\varphi \quad, \Psi=\pi / 2
$$

Thus:

$$
R_{\phi}=\left[\begin{array}{cccc}
1 & 0 & 0 & 0 \\
0 & 1 & 0 & 0 \\
0 & 0 & \cos \varphi & \sin \varphi \\
0 & 0 & -\sin \varphi & \cos \varphi
\end{array}\right], S_{\varphi}=\left[\begin{array}{cc}
\cos \varphi / 2 & -i \sin \varphi / 2 \\
i \sin \varphi / 2 & \cos \varphi / 2
\end{array}\right]
$$

If the vector $\mathbf{q}$ has components $q(0, \cos \varphi, \sin \varphi)$ in the coordinate system $x, y, z$, then the wave function can be written in the form:

$$
\psi_{\mathbf{q}} \equiv \psi_{\varphi}=D_{-\varphi} \psi_{0}
$$

The previously found wave function has a zero subscript. The wave function of heavy holes, found using (A6), is written below, as it will be used later.

$$
\begin{aligned}
\boldsymbol{\psi}_{h}(q, x, \phi) & =H_{1}\left[\begin{array}{c}
q \cos k_{h} x e^{-i \phi} \xi \\
-i k_{h} \sin k_{h} x \xi-q \cos k_{h} x \sin \phi \eta \\
-k_{h} \sin k_{h} x \xi+q \cos k_{h} x \cos \phi \eta
\end{array}\right]+ \\
& +H_{2}\left[\begin{array}{c}
q \sin k_{h} x e^{i \phi} \eta \\
i k_{h} \cos k_{h} x \eta+q \sin k_{h} x \sin \phi \xi \\
-k_{h} \cos k_{h} x \eta-q \sin k_{h} x \cos \phi \xi
\end{array}\right]
\end{aligned}
$$

The boundary conditions for hole wave functions can be derived by integrating Kane's equations across the heteroboundary (see Section II, paragraph 1.C). We consider generalized Luttinger parameters $\tilde{\gamma}_{1}$ and $\tilde{\gamma}_{2}$ also to be continuous for the sake of simplicity. Taking into account that $m_{l}^{-1} \approx \frac{2 \gamma^{2}}{E_{g}+\delta-E} \gg m_{h}^{-1}$ we obtain continuity conditions at the heteroboundary for the following quantities:

1) $\psi$

2) $\frac{\partial}{\partial x} \boldsymbol{\psi}_{\perp}$,

3) $\frac{1}{E_{g}+\delta-E} \operatorname{div} \boldsymbol{\psi}$. 
Generally speaking the wave functions of holes in a quantum well are superposition of three subbands of the valence band: of heavy, light, and SO holes. However, the last subband strongly, exponentially, decays away from the heteroboundary with a exponent $\kappa_{s o} \approx \sqrt{\frac{m_{h} \Delta}{\hbar^{2}} \frac{4}{3}}$. As a consequence, this branch mainly affects the derivative of the wave function near the heteroboundary, and its influence on the wave function itself is negligible. It should be emphasized that such an approximation is not equivalent to using the $4 \times 4$ Hamiltonian from the very beginning. We shall seek the wave function as a superposition of the heavy- and light-hole subbands. Near the upper edge of the valence band the parameter $\left|\lambda_{s o}\right| \approx \frac{m_{h}}{m_{l}} \gg 1$. This means that only the first and the third of the boundary conditions (A8) are applicable. In this approximation, light and heavy holes do not mix with each other and have different spectra. The heavy hole spectrum coincides with the quantum mechanical spectrum of a particle in a rectangular quantum well. For states with even and odd $\mid x>$-components of the wave function of heavy holes the dispersion equations are following:

$$
\begin{aligned}
& \tan k_{h} a / 2=\frac{\kappa_{h}}{k_{h}}-\text { for even states } \\
& \cot k_{h} a / 2=-\frac{k_{h}}{\kappa_{h}}-\text { for odd states }
\end{aligned}
$$

For light holes the states with different parities cannot be separated, and the dispersion equation becomes more cumbersome:

$$
\begin{aligned}
& {\left[\frac{E_{g}+\delta+V_{c}-E}{E_{g}+\delta-E} \frac{k_{l}^{2}+q^{2}}{\kappa_{l}^{2}-q^{2}} \kappa_{l} \cot k_{l} a / 2+k_{l} \frac{2 \lambda_{l}-1}{2 \tilde{\lambda}_{l}-1}\right] \times } \\
\times & {\left[\frac{E_{g}+\tilde{\delta}+V_{c}-E}{E_{g}+\delta-E} \frac{k_{l}^{2}+q^{2}}{\kappa_{l}^{2}-q^{2}} \kappa_{l} \tan k_{l} a / 2-k_{l} \frac{2 \lambda_{l}-1}{2 \tilde{\lambda}_{l}-1}\right]=} \\
= & q^{2}\left[\frac{2 \lambda_{l}-1}{2 \tilde{\lambda}_{l}-1}+\frac{E_{g}+\tilde{\delta}+V_{c}-E}{E_{g}+\delta-E} \frac{k_{l}^{2}+q^{2}}{\kappa_{l}^{2}-q^{2}}\right]^{2} .
\end{aligned}
$$

Here $\kappa_{l}$ and $\kappa_{h}$ denote the moduli of $\mathrm{x}$ quasi-momentum components of light and heavy holes in the barrier region respectively,

$$
\tilde{\lambda}_{i}=\frac{\tilde{\delta}}{U_{v}+E+2 \tilde{\delta}+\hbar^{2} \kappa_{l}^{2} / 2 m_{h}}, \quad \tilde{\delta}=\frac{\tilde{\Delta_{s o}}}{3} .
$$

Note that at $q=0$ the light hole states are also split into states with different parities. The constants $H_{i}$ and $L_{i}$ in (A1), (A2) are determined by normalization conditions. In particular:

$$
H_{i}=\frac{1}{\sqrt{q^{2}+k_{h}^{2}}} \frac{1}{\sqrt{a+\frac{1}{\kappa_{h}} \frac{q^{2}}{q^{2}+k_{h}^{2}}}} .
$$

The opposite is the case for SO holes. The components of the wave functions of light and heavy holes oscillate rapidly, and the contribution from them to overlap integrals is negligibly 
small. Similarly, it is easy to verify that for the $s o$-component $\psi_{x}$ and $\operatorname{div} \psi /\left(E_{g}+\delta-E\right)$ are to be considered continuous. The type of wave functions of SO holes is similar to that for the light holes (A2), (A3). Strictly speaking, with the condition $E_{g}-\Delta>U_{v}$ fulfilled the spectrum of spin-split holes is continuous. However, when the rapidly oscillating contributions from the subbands of light and heavy holes are neglected, the spectrum may be both continuous and discrete. In the general case, near such a quasi-discrete level there exists a peak of density of states with small hole momenta in the direction perpendicular to the heteroboundary. The spectrum of holes of this kind is similar to that of light holes. The corresponding dispersion equation for SO holes has the form

$$
\begin{aligned}
& {\left[\frac{E_{g}+\tilde{\delta}+V_{c}-E}{E_{g}+\delta-E} \frac{k_{s o}^{2}+q^{2}}{\kappa_{s o}^{2}-q^{2}} \kappa_{s o} \cot k_{s o} a / 2+k_{s o}\right] \times } \\
\times & {\left[\frac{E_{g}+\tilde{\delta}+V_{c}-E}{E_{g}+\delta-E} \frac{k_{s o}^{2}+q^{2}}{\kappa_{s o}^{2}-q^{2}} \kappa_{s o} \tan k_{s o} a / 2-k_{s o}\right]=} \\
= & q^{2}\left[\frac{\lambda_{s o}}{\tilde{\lambda}_{s o}}+\frac{E_{g}+\tilde{\delta}+V_{c}-E}{E_{g}+\delta-E} \frac{k_{s o}^{2}+q^{2}}{\kappa_{s o}^{2}-q^{2}}\right]^{2} .
\end{aligned}
$$

\section{Electrons}

Electrons obey the same symmetry rules as holes. Their wave functions are similar to those of light holes and can be written as:

$$
\begin{aligned}
& \text { at }|x|<a / 2 \\
& \psi_{s c}=A_{1} \cos k_{c} x \eta+A_{2} \sin k_{c} x \xi \\
& \psi_{c}=\frac{i \hbar \gamma}{Z} A_{1}\left(\begin{array}{c}
k_{c} \sin k_{c} x \eta-\lambda_{c} q \cos k_{c} x \xi \\
-i q \cos k_{c} x \eta+i \lambda_{c} k_{c} \sin k_{c} x \xi \\
-\lambda_{c} k_{c} \sin k_{c} x \xi+\lambda_{c} q \cos k_{c} x \eta
\end{array}\right)+ \\
& +\frac{i \hbar \gamma}{Z} A_{2}\left(\begin{array}{c}
-k_{c} \cos k_{c} x \xi-\lambda_{c} q \sin k_{c} x \eta \\
-i \lambda_{c} k_{c} \cos k_{c} x \eta-i q \sin k_{c} x \xi \\
-\lambda_{c} q \sin k_{c} x \xi-\lambda_{c} k_{c} \cos k_{c} x \eta
\end{array}\right)
\end{aligned}
$$

where

$$
\begin{aligned}
Z & =\frac{\mathcal{E}^{2}+\mathcal{E}\left(2 E_{g}+2 \delta\right)+\left(E_{g}+3 \delta\right) E_{g}}{\mathcal{E}+E_{g}+2 \delta} \\
\lambda_{c} & =\frac{\delta}{\mathcal{E}+E_{g}+2 \delta} .
\end{aligned}
$$


Here $k_{c}$ denotes the $x$-component of the quasi-momentum of electrons in a quantum well, $q$ is the longitudinal quasi-momentum of electrons. Functions with another symmetry can be derived by the same procedure as that used for holes. From the boundary condition follows that $\psi_{s}$ and $\psi_{x}$ must be continuous. This yields the following dispersion equation:

$$
\left(k_{c} \tan k_{c} a / 2-\frac{Z}{\tilde{Z}} \kappa_{c}\right)\left(k_{c} \cot k_{c} a / 2+\frac{Z}{\tilde{Z}} \kappa_{c}\right)=-q^{2}\left(\lambda_{c}-\tilde{\lambda_{c}} \frac{Z}{\tilde{Z}}\right)^{2},
$$

where $\kappa_{c}$ is the modulus of the $x$ quasi-momentum component of electrons in the barrier region,

$$
\begin{aligned}
& \tilde{Z}=\frac{\mathcal{E}^{2}+\mathcal{E}\left(2 E_{g}+2 U_{v}+2 \tilde{\delta}\right)+\left(E_{g}+U_{v}+3 \tilde{\delta}\right)\left(E_{g}+U_{v}\right)}{\mathcal{E}+E_{g}+U_{v}+2 \tilde{\delta}} \\
& \tilde{\lambda_{c}}=\frac{\tilde{\delta}}{\mathcal{E}+E_{g}+U_{v}+2 \tilde{\delta}} .
\end{aligned}
$$

The spectrum splits into even and odd states if the longitudinal wave vector $(q)$ is small or the expression in parentheses in the right-hand side of the equation is close to zero. The last condition is commonly fulfilled, since, as a rule, $U_{v} \ll E_{g}$, which corresponds to semiconductors with about the same band structure. Note, that in the case of the discontinuous Kane parameter $\gamma \neq$ const, continuity of $\gamma \psi_{x}$ and $\psi_{s}$ should be used 9 .

\section{APPENDIX B: COULOMB POTENTIAL IN THE PRESENCE OF HETEROBOUNDARIES}

In a quantum well the Coulomb potential of a charged particle differs from that in a homogeneous semiconductor, because of the different dielectric constants in a quantum well and barrier region:

$$
\Phi\left(\mathbf{r}_{0}, \mathbf{r}\right)=\frac{e}{\kappa_{0}\left|\mathbf{r}-\mathbf{r}_{0}\right|}+\tilde{\Phi}\left(\mathbf{r}_{0}, \mathbf{r}\right),
$$

where $\mathbf{r}_{0}$ is the coordinate of the particle and $\mathbf{r}$ is the coordinate of the point where the potential is observed. We consider only the case, when the particle is inside the quantum well $\left(\left|x_{0}\right|<a / 2\right)$. Using the reflection method (see e.g. 34) one can obtain:

$$
\begin{aligned}
\tilde{\Phi} & =\sum_{n \geq 1} \frac{e}{\kappa_{0}}\left(\frac{\kappa_{0}-\tilde{\kappa}_{0}}{\kappa_{0}+\tilde{\kappa}_{0}}\right)^{2 n-1}\left(\frac{1}{\sqrt{\left(x+x_{0}-(2 n-1) a\right)^{2}+\rho^{2}}}+\frac{1}{\sqrt{\left(x+x_{0}+(2 n-1) a\right)^{2}+\rho^{2}}}\right)+ \\
& +\sum_{n \geq 1} \frac{e}{\kappa_{0}}\left(\frac{\kappa_{0}-\tilde{\kappa}_{0}}{\kappa_{0}+\tilde{\kappa}_{0}}\right)^{2 n}\left(\frac{1}{\sqrt{\left(x-x_{0}-2 n a\right)^{2}+\rho^{2}}}+\frac{1}{\sqrt{\left(x-x_{0}+2 n a\right)^{2}+\rho^{2}}}\right) \text { at }|x|<a / 2 \\
\tilde{\Phi} & =\frac{e}{\kappa_{0} \sqrt{\left(x-x_{0}\right)^{2}+\rho^{2}}} \frac{\kappa_{0}-\tilde{\kappa}_{0}}{\kappa_{0}+\tilde{\kappa}_{0}}+\frac{2 e}{\kappa_{0}+\tilde{\kappa}_{0}} \sum_{n \geq 1}\left(\frac{\kappa_{0}-\tilde{\kappa}_{0}}{\kappa_{0}+\tilde{\kappa}_{0}}\right)^{2 n} \frac{1}{\sqrt{\left(x-x_{0}+2 n a\right)^{2}+\rho^{2}}}+ \\
& +\frac{2 e}{\kappa_{0}+\tilde{\kappa}_{0}} \sum_{n \geq 1}\left(\frac{\kappa_{0}-\tilde{\kappa}_{0}}{\kappa_{0}+\tilde{\kappa}_{0}}\right)^{2 n-1} \frac{1}{\sqrt{\left(x+x_{0}+(2 n-1) a\right)^{2}+\rho^{2}}} \text { at } x>a / 2 .
\end{aligned}
$$


Here $\rho^{2}=\left(y-y_{0}\right)^{2}+\left(z-z_{0}\right)^{2}$, $a$ is a quantum well width. These potentials are rather cumbersome. However, they may be simplified if dielectric constants $\kappa_{0}$ and $\tilde{\kappa}_{0}$ are supposed to be close to each other. After taking Fourier transforming along lateral coordinates ( $y$ and $z$ ) one obtains:

$$
\begin{aligned}
& \phi(x, q) \approx \frac{e}{2 q \kappa_{0}}\left(e^{-q\left|x-x_{0}\right|}+2 \frac{\kappa_{0}-\tilde{\kappa}_{0}}{\kappa_{0}+\tilde{\kappa}_{0}} \operatorname{ch}\left(q\left(x+x_{0}\right)\right) e^{-q a}\right) \text { at }|x|<a / 2 \\
& \phi(x, q) \approx \frac{e}{q\left(\kappa_{0}+\tilde{\kappa}_{0}\right)}\left(e^{-q\left(x-x_{0}\right)}+\frac{\kappa_{0}-\tilde{\kappa}_{0}}{\kappa_{0}+\tilde{\kappa}_{0}} e^{-q\left(x+x_{0}+a\right)}\right) \text { at } x>a / 2 .
\end{aligned}
$$

One may see that while potential itself is a continuous function along the interface, difference between its left and right derivatives is proportional to $\left(\kappa_{0}-\tilde{\kappa}_{0}\right) /\left(\kappa_{0}+\tilde{\kappa}_{0}\right)$. 


\section{REFERENCES}

${ }^{1}$ A.R. Beattie and P.T. Landsberg, Proc. Roy. Soc., A 249, 16 (1959).

${ }^{2}$ B.L. Gel'mont, Sov. Phys. JETP, 48, 268 (1978).

${ }^{3}$ A. Haug, J. Phys. C: Solid State Phys., 16, 4159 (1983).

${ }^{4}$ M. Takeshima, Phys. Rev., B 28, 2039 (1983).

${ }^{5}$ G. P. Agrawal and N. K. Dutta. Long-Wavelength Semiconductor Lasers. Van Nostrand Reinhold, New York (1993).

${ }^{6}$ M. Aidaraliev et al., Sov. Phys. Semicond. 26, 138 (1992).

${ }^{7}$ G.G. Zegrya and V.A. Kharchenko, Sov. Phys. JETP, 74, 173 (1992).

${ }^{8}$ Quantum Well Lasers, Edited by Peter S. Zory, Jr., Academic Press, Inc., 1993.

${ }^{9}$ R.I. Taylor et al., IEE Proceedings 132, 364 (1985).

${ }^{10}$ W.W. Lui et al., Phys. Rev. B 48, 8814 (1993).

${ }^{11}$ N.K. Dutta, J. Appl. Phys. 54, 1236 (1983)

${ }^{12}$ G.G. Zegrya et al., 23rd Intern. Symposium on Compound Semiconductors (ISCS-23), St.Petersburg, Russia, 23-27 September, p. 122 (1996).

${ }^{13}$ R.I. Taylor et al., Semicond. Sci. Technol., 5, 90 (1990)

${ }^{14}$ M.I. Dyakonov and V.Yu. Kachorovskii, Phys. Rev., B 49, 17130 (1994).

${ }^{15}$ G.G. Zegrya et al., Proc. SPIE, 2399, 307 (1995).

${ }^{16}$ E.O. Kane, J. Phys. Chem. Sol., 1, 249 (1957).

${ }^{17}$ P.C. Sercel and K.J. Vahala, Phys. Rev., B 42, 3690 (1990).

${ }^{18}$ R.A. Suris, Sov. Phys. Semicond., 20, 1258 (1986).

${ }^{19}$ M.G. Burt, J. Phys.: Condens. Matter, 4, 6651 (1992).

${ }^{20}$ B.A.Foreman, Phys. Rev., B 49, 1757 (1994).

${ }^{21}$ G.L. Bir and G.E. Pikus. Symmetry and Strain-Induced Effects in Semiconductors (Willey, New York, 1974).

${ }^{22}$ L.D. Landau and E.M. Lifshitz, Quantum Mechanics (Pergamon New York, 1977). 
${ }^{23}$ J. Wang, P. von Allmen, J.-P. Leburton, and K.J. Linden, IEEE J. Quantum Electron. QE31, 864 (1995).

${ }^{24}$ A. Haug, J. Phys. Chem. Solids, 49, 599 (1988).

${ }^{25}$ G.G. Zegrya and A.S.Polkovnikov to be published.

${ }^{26}$ G.G. Zegrya and A.S. Polkovnikov, JETP, accepted to publication (1998).

${ }^{27}$ A. Haug, Appl. Phys. A 51, 354 (1990)

${ }^{28}$ B.K. Ridley, J. Phys. C: Solid State Phys., 15, 5899 (1982).

${ }^{29}$ G.G. Zegrya. Antimonide Related Strained Layer Heterostructures, M.O. Manasreh (ed.), Gordon and Breach, Neward (1997).

${ }^{30}$ S. Ideshita et al., Appl. Phys. Lett. 60, 25941992

${ }^{31}$ M. Sweeny and J. Xu, Appl. Phys. Lett. 54, 546 (1989)

${ }^{32}$ E.P. O'Reilly and M. Silver, Appl. Phys. Lett., 63, 3318 (1993).

${ }^{33}$ E.P. O'Reilly and A.R. Adams, IEEE J. Quantum Electron. 30, 366 (1994).

${ }^{34}$ L.D. Landau and E.M. Lifshitz, Electrodynamics of Continuous Media (Pergamon New York, 1977). 


\section{Footnotes}

1. We assume that, as is commonly the case, $\left(V_{c}, V_{v}\right)<E_{g}$

2. The reason is that this term has the lowest threshold energy. By the threshold energy we understand the mean energy of a heavy hole taking part in an Auger transition 


\section{Figure Captions}

Fig. 1 Auger transition probabilities $w_{1}(q)$ and $w_{2}(q)$, corresponding to the thresholdless and quasi-threshold matrix elements $M_{1}$ and $M_{2}$ as functions of the longitudinal momentum of heavy holes at $T=300 K$ for different quantum well widths ((a) $a=50 \AA$, (b) $a=100 \AA$, (c) $a=200 \AA$, and (d) $a=500 \AA$ ). The model parameters typical of InGaAsP/InP quantum wells with $E_{g} \approx 1 \mathrm{eV}$ were used.

Fig.2 Threshold energy of the CHCC process as a function of the quantum well width for three mechanisms of Auger recombination: thresholdless $\left(E_{t h}^{1}\right)$, quasi-threshold $\left(E_{t h}^{2}\right)$, and threshold $\left(E_{t h}^{3}\right)$ at $T=300 K$. The solid curve corresponds to the threshold energy $\left(E_{t h}^{t o t}\right)$ of the total Auger coefficient $\left(C=C_{1}+C_{2}+C_{3}\right)$. The horizontal dashed line corresponds to the threshold energy $E_{t h}^{3 D}$ for the bulk Auger process.

Fig. 3 Auger coefficients $C_{1}$ and $C_{2}$ for the thresholdless and quasi-threshold processes as functions of a quantum well width at different temperatures ((a) $T=50 K$, (b) $T=150 K$, (c) $T=300 K$, and (d) $T=400 K)$. The same parameters as in Fig. 1 were used

Fig. 4 Coefficient $C_{3}$ for the threshold Auger process as a function of the quantum well width at $T=150 K(\mathrm{a})$ and $T=300 K(\mathrm{~b})$.

Fig. 5 Temperature dependence of the total Auger coefficient and the partial contributions of the thresholdless and quasi-threshold mechanisms at different quantum well widths. The same parameters were used as in Fig. 1.

Fig. 6 Comparison of the thresholdless Auger coefficient $\left(C_{1}\right)$ as a function of temperature at various Fermi energies of the holes for two different quantum well thicknesses ((a) $a=50 \AA$ and (b) $a=500 \AA$ ). $T_{F}$ denotes the Fermi energy expressed in Kelvins. The curve with $T_{F}=-100 \mathrm{~K}$ approximately corresponds to the Boltzmann statistics.

Fig. 7 Phonon-assisted Auger coefficients corresponding to the thresholdless and quasithreshold matrix elements as functions of temperature at different quantum well widths ((a) $a=50 \AA$ and (b) $a=150 \AA$ ).

Fig. 8 Three-dimensional Auger coefficients $C_{1} a^{2}$ and $\left(C_{2}+C_{3}\right) a^{2}$ as functions of a quantum well width at $T=300 \mathrm{~K}$. The same parameters as in Fig. 1 were used. 

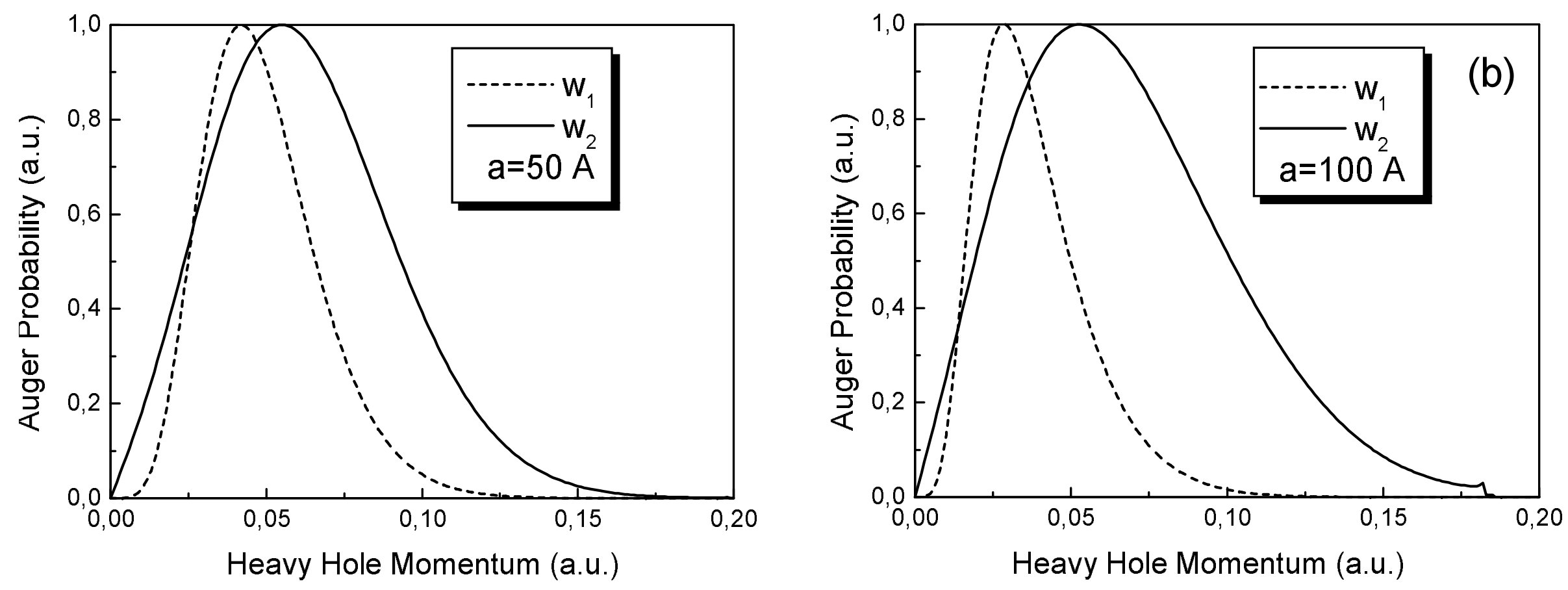

Fig. 1
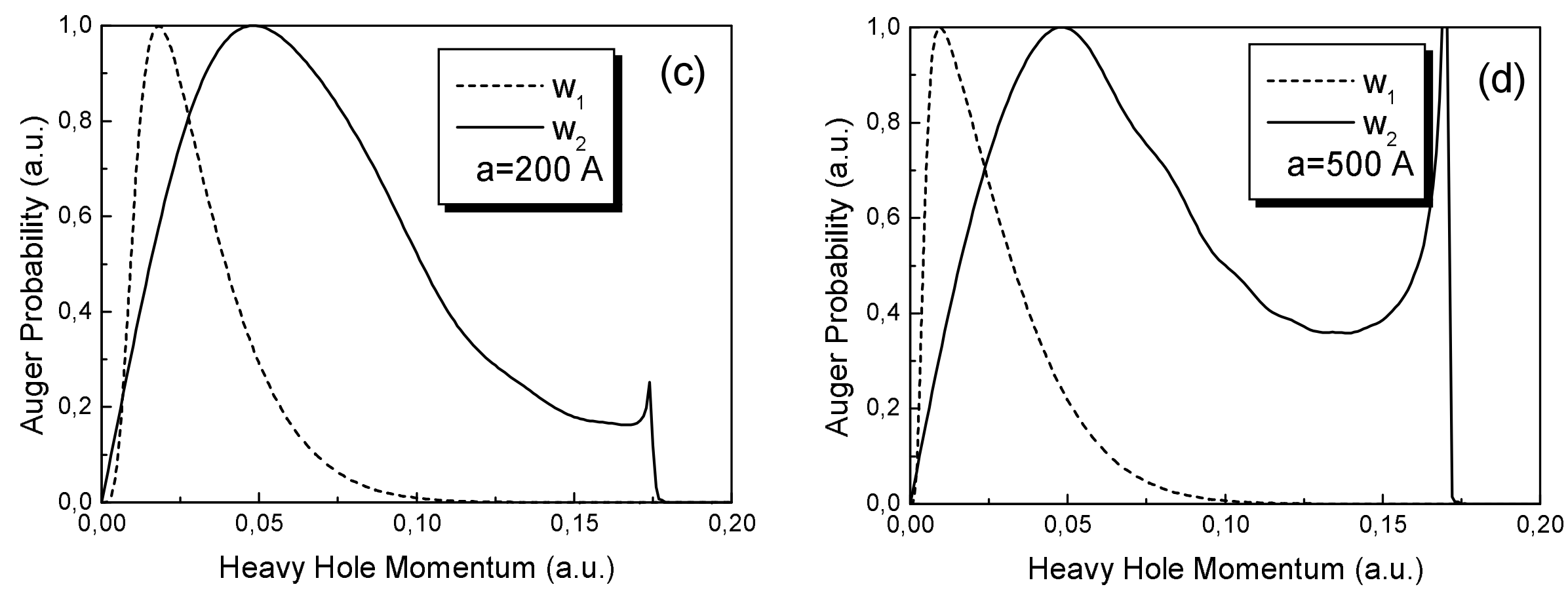
Fig. 2

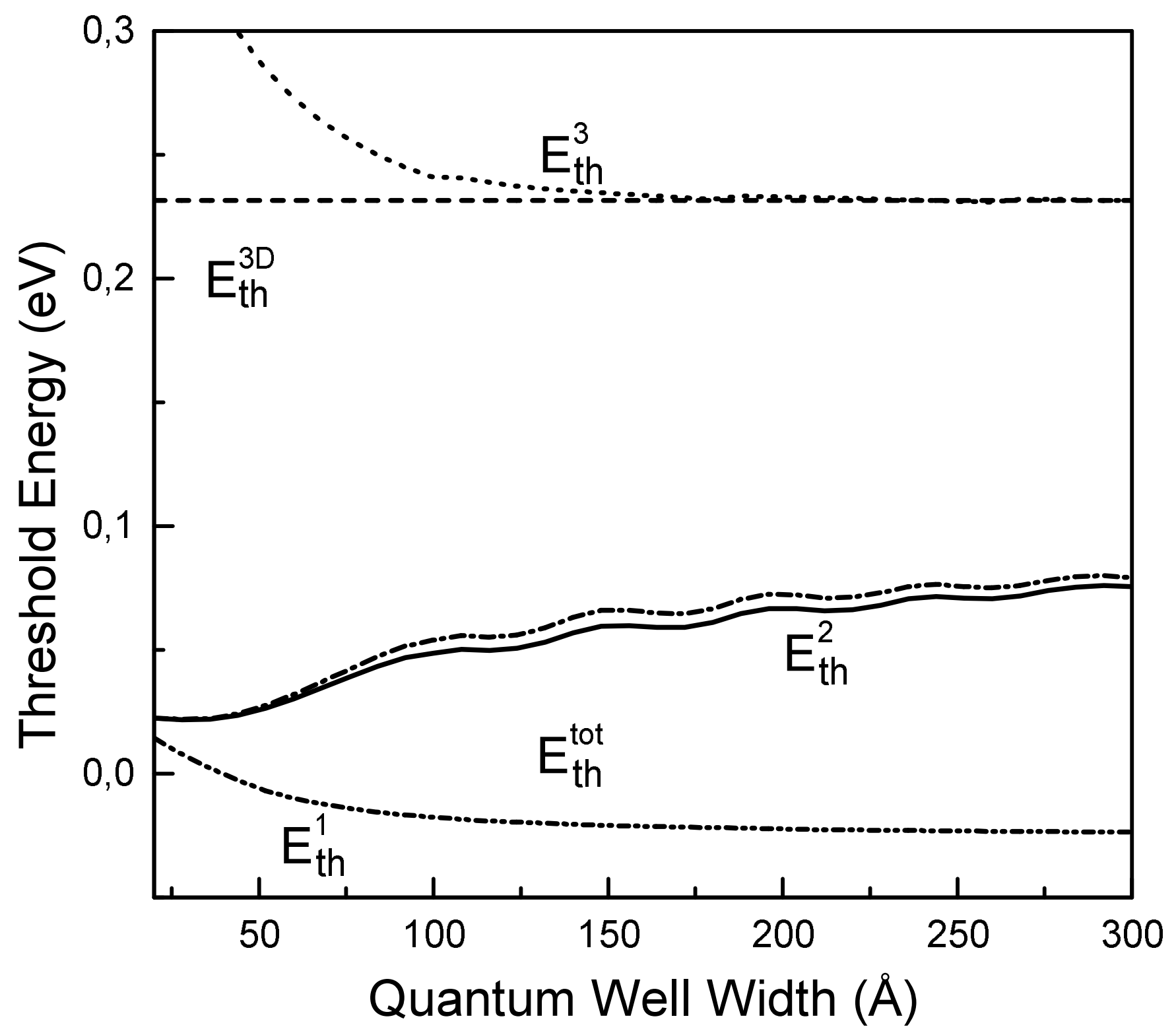




\section{Auger Coefficients \\ Fig. 3}
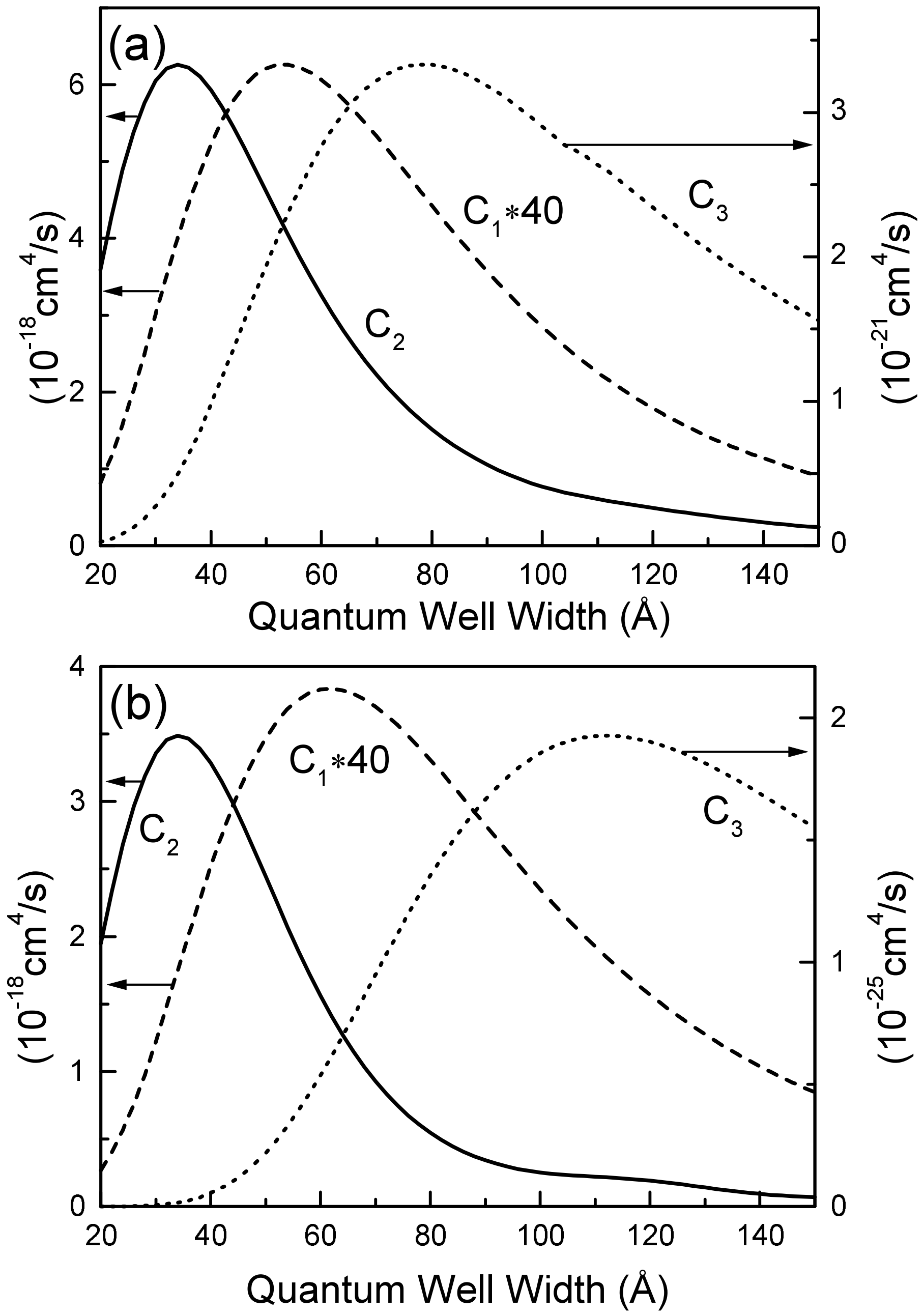

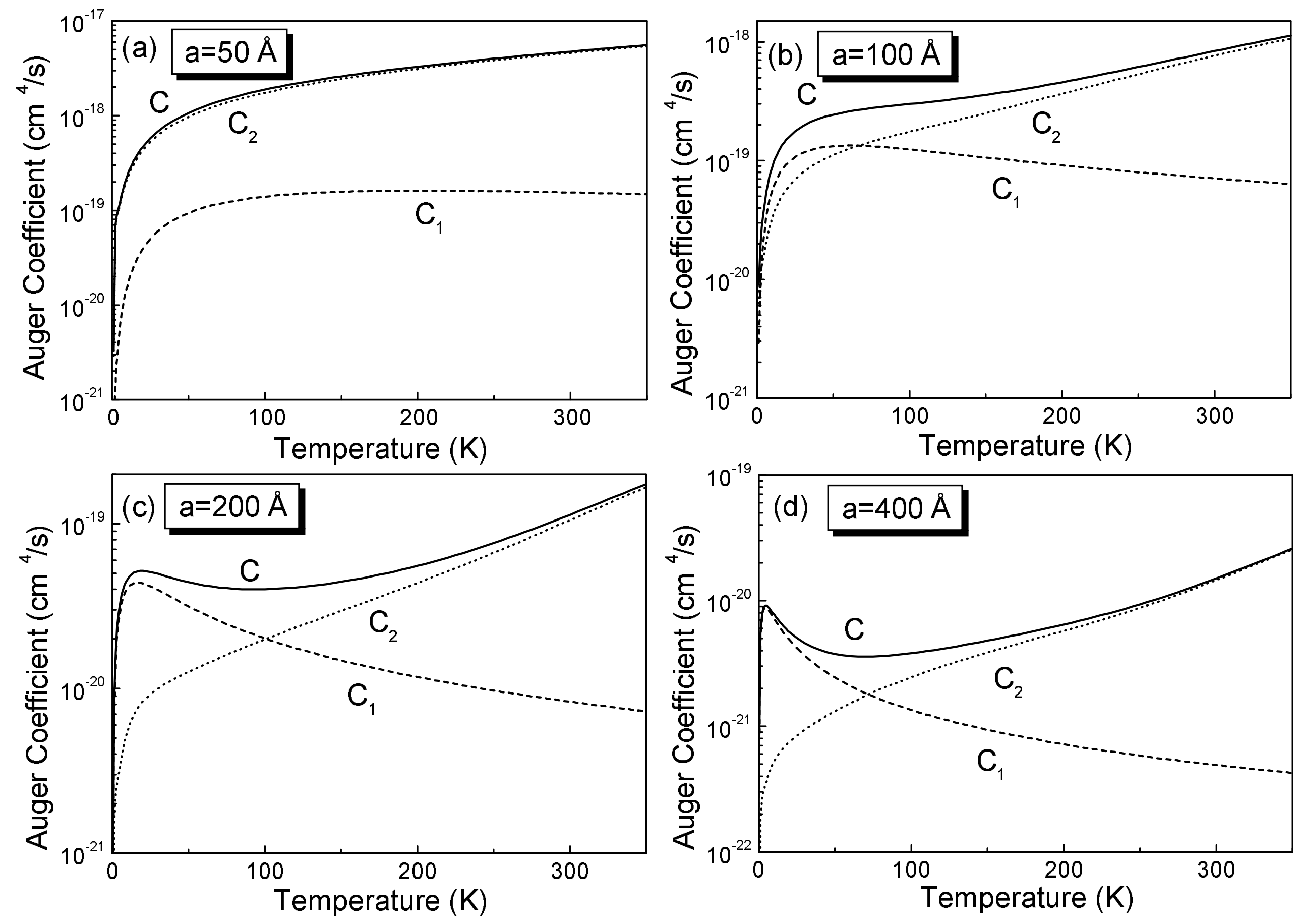
Fig. 5
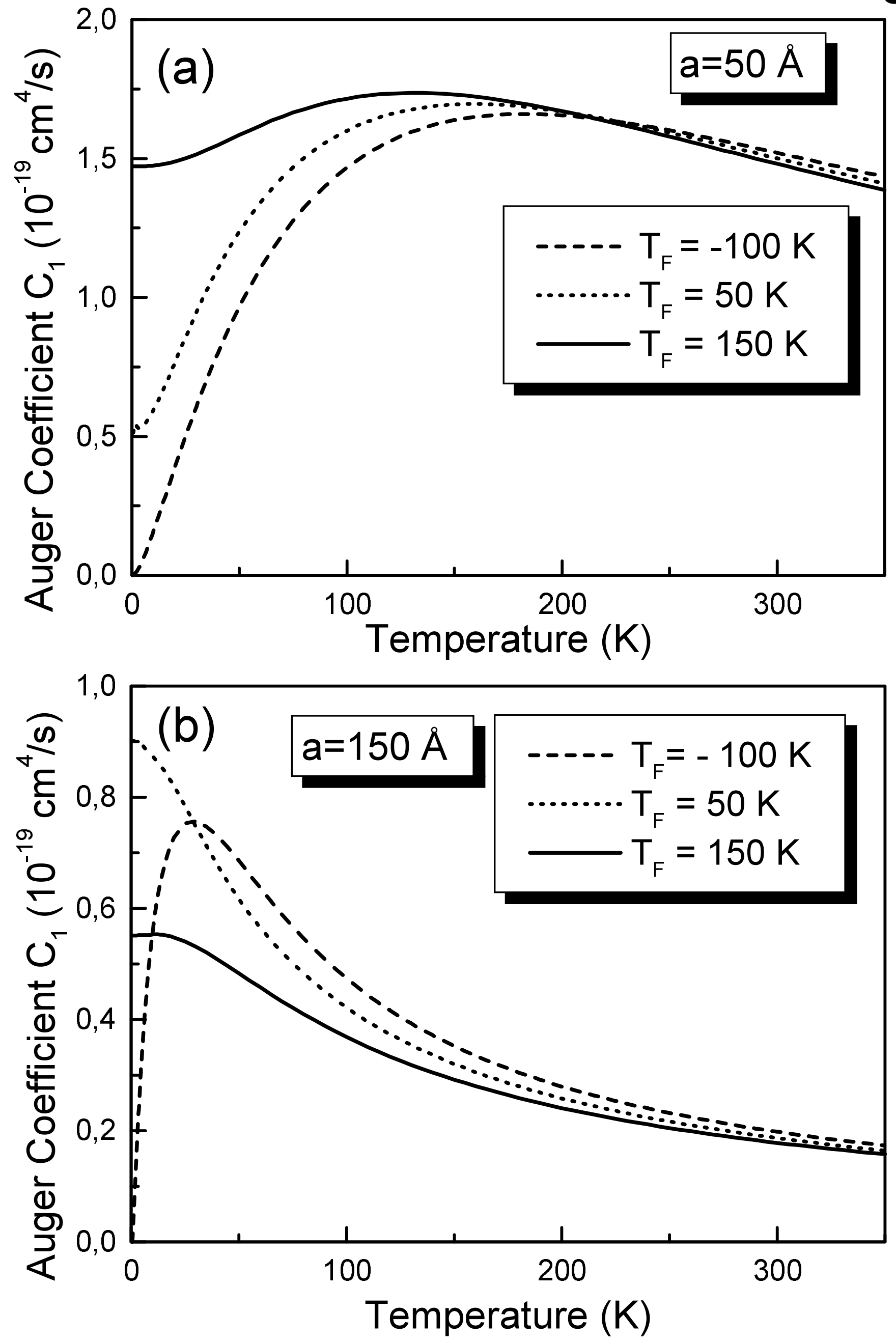
Fig. 6
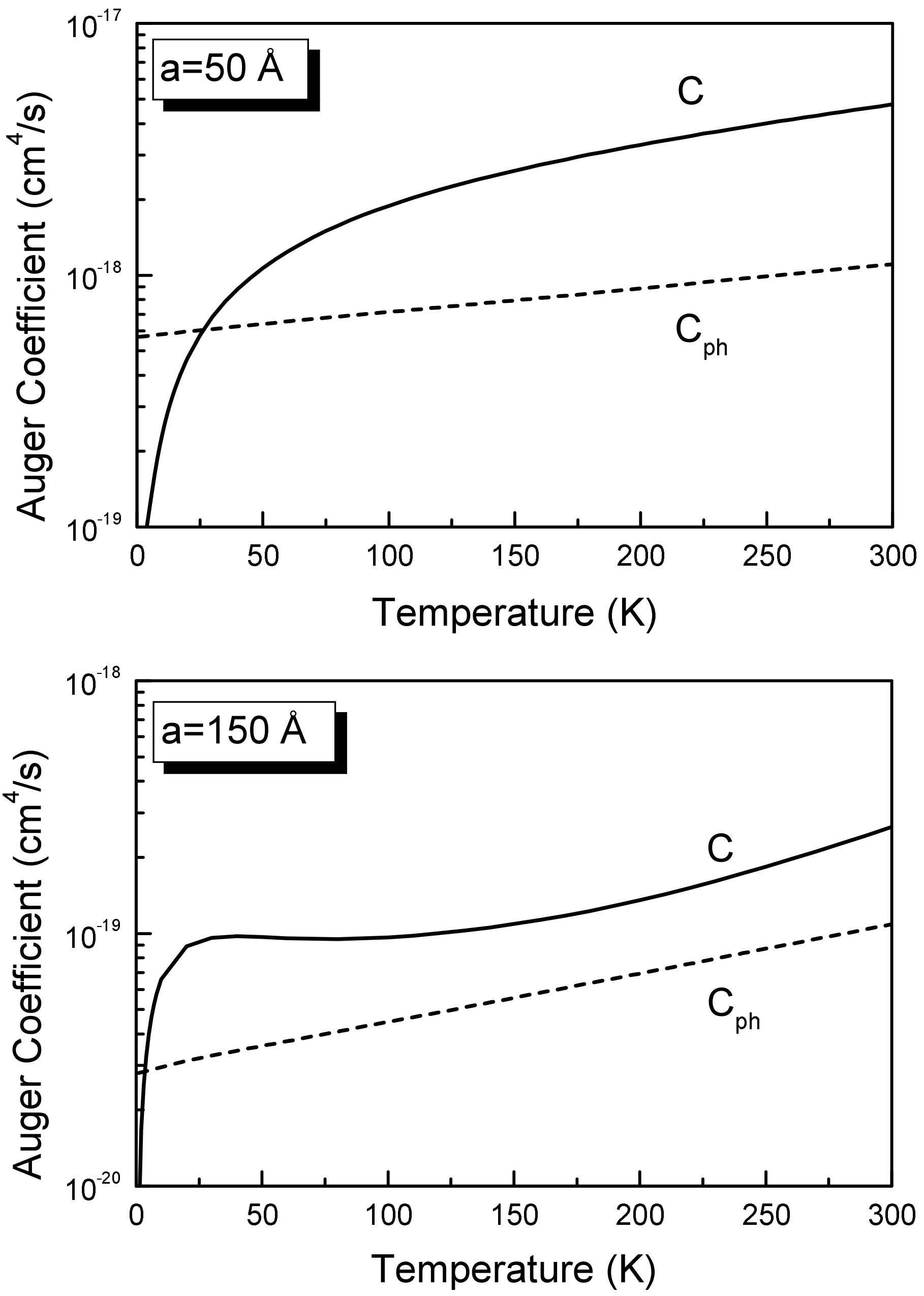
Fig. 7

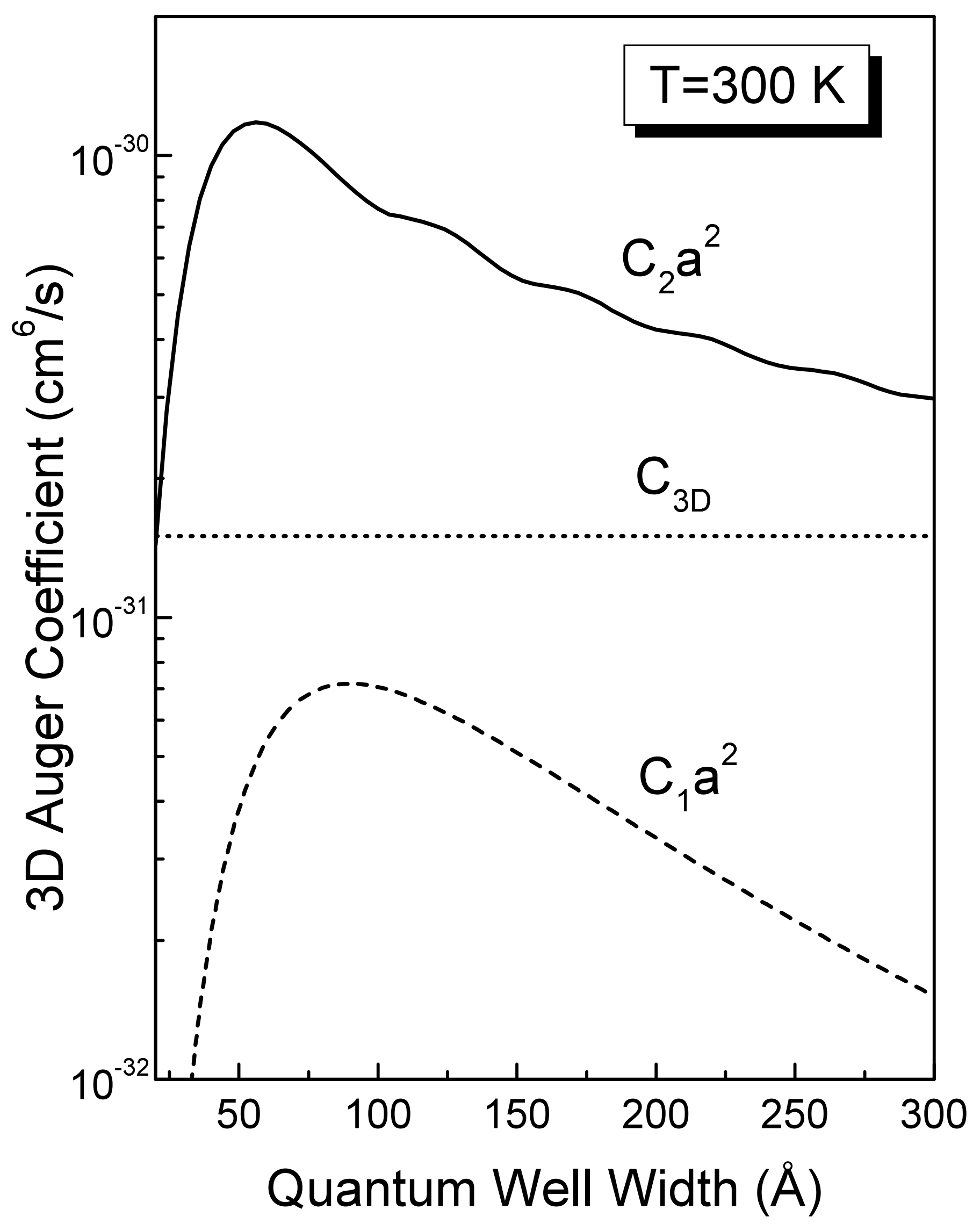

